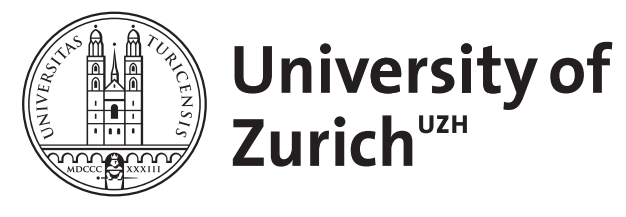

\title{
Genetic approaches to retinal research in zebrafish
}

Niklaus, Stephanie ; Neuhauss, Stephan C F

\begin{abstract}
The zebrafish (Danio rerio) possesses a vertebrate-type retina that is extraordinarily conserved in evolution. This well-organized and anatomically easily accessible part of the central nervous system has been widely investigated in zebrafish, promoting general understanding of retinal development, morphology, function and associated diseases. Over the recent years, genome and protein engineering as well as imaging techniques have experienced revolutionary advances and innovations, creating new possibilities and methods to study zebrafish development and function. In this review, we focus on some of these emerging technologies and how they may impact retinal research in the future. We place an emphasis on genetic techniques, such as transgenic approaches and the revolutionizing new possibilities in genome editing.
\end{abstract}

DOI: https://doi.org/10.1080/01677063.2017.1343316

Posted at the Zurich Open Repository and Archive, University of Zurich

ZORA URL: https://doi.org/10.5167/uzh-139441

Journal Article

Accepted Version

Originally published at:

Niklaus, Stephanie; Neuhauss, Stephan C F (2017). Genetic approaches to retinal research in zebrafish. Journal of Neurogenetics, 31(3):70-87.

DOI: https://doi.org/10.1080/01677063.2017.1343316 


\section{Genetic Approaches to Retinal Research in Zebrafish}

Stephanie Niklaus ${ }^{1,2}$ \& Stephan C.F. Neuhauss ${ }^{1 \S}$

University of Zurich, Institute of Molecular Life Sciences ${ }^{1}$, Life Science Zürich Graduate Program Neuroscience ${ }^{2}$, Winterthurerstrasse 190, CH-8057 Zurich, Switzerland

${ }^{\S}$ Corresponding author:

Prof. Dr. Stephan C. F. Neuhauss

Institute of Molecular Life Sciences

University of Zurich

Winterthurerstrasse 190

$\mathrm{CH}-8057$ Zurich, Switzerland

stephan.neuhauss@imls.uzh.ch 


\begin{abstract}
The zebrafish (Danio rerio) possesses a vertebrate type retina that is extraordinarily conserved in evolution. This well organized and anatomically easily accessible part of the central nervous system has been widely investigated in zebrafish, promoting general understanding of retinal development, morphology, function and associated diseases.
\end{abstract}

Over the recent years, genome and protein engineering as well as imaging techniques have experienced revolutionary advances and innovations, creating new possibilities and methods to study zebrafish development and function. In this review we focus on some of these emerging technologies and how they may impact retinal research in the future. We place an emphasis on genetic techniques, such as transgenic approaches and the revolutionizing new possibilities in genome editing.

\title{
INTRODUCTION
}

In recent years, the small zebrafish (Danio rerio) made a big splash as a model for vertebrate biology in general and nervous system development in particular. This small tropical teleost fish owes this honor mainly due to its favorable biological properties, combining many advantages of simple invertebrate models with the ones of more complex vertebrates. It possesses a canonical vertebrate nervous system, with evolutionarily conserved anatomical subdivisions, cell types, receptors, channels and neurotransmitter systems. But it also possesses attributes more commonly found in invertebrates, such as small body size, ease of maintenance and breeding, high number of offspring (one pair can produce up to 200 progeny per week) and more compact neuronal circuits. Additional points in favor of 
zebrafish are its rapid extra-corporal development and transparency of offspring, making them ideally suited for live imaging.

The main reason for the zebrafish to join the exclusive club of the few most studied biomedical model species lies in its superb genetics with continuous additions to its genetic toolbox. The zebrafish genome is by now fully sequenced and annotated (Howe et al. 2013) and for more than $70 \%$ of human genes at least one zebrafish ortholog can be identified (Howe et al. 2013). Therefore human genetic diseases can readily be modeled in zebrafish.

Recent years saw a massive expansion of the genetic toolbox to manipulate zebrafish. Transposon mediated transgenesis allows efficient insertion of DNA cassettes into the zebrafish genome. Spatial and temporal control of transgene expression can now be achieved through the use of various cell and tissue specific and inducible regulatory elements.

Finally the field of genome editing has seen a revolution with the introduction of the CRISPR/Cas system, enabling easy and efficient site-directed mutagenesis in zebrafish.

Equipped with this vast and growing array of tools, the vertebrate retina can be effectively studied in zebrafish. The zebrafish retina develops early on in development and shows all the features of a canonical vertebrate retina already 5 days post fertilization (dpf).

Due to the diurnal life style of the zebrafish, its retina supports daylight vision and is therefore in many aspects closer related to the human retina than the retina of mostly nocturnal rodents. 
In the following section we will briefly outline the structure and functionality of the zebrafish retina, before embarking on a description of modern genetic tools that became recently available for the study of the zebrafish retina.

\section{The Zebrafish Retina}

The zebrafish possesses a canonical vertebrate retina that is amenable to a detailed analysis of its development and functional output by various electrophysiological and behavioral means. As vision is the primary sense at larval stages used for prey capture and predator avoidance, strong selective pressure has been placed on the rapid development of the retina and downstream visual processing centers in the rest of the brain.

\section{Retinal Anatomy}

The zebrafish retina is a canonical vertebrate retina with the neural retina consisting of three nuclear layers, separated by two synaptic layers. As in all vertebrate retinas there are five major neuronal and one glial cell type stereotypically situated in these nuclear layers. The outer nuclear layer is made up of the nuclei of photoreceptors. The inner nuclear layer is composed of cell bodies of horizontal, bipolar, amacrine, and Müller glia cells. Finally the ganglion cell layer mainly consists of ganglion cell nuclei, but also of nuclei of displaced amacrine cells (Figure 1).

This remarkably ordered array is already present in the early larva at $5 \mathrm{dpf}$ (Figure 1a).

These major cell types can be further subdivided into numerous subtypes, by both morphological and physiological criteria. The exact number of these subtypes varies between different vertebrates, likely reflecting adaptation to differing visual ecologies, and is controversially debated in most species. 
The zebrafish outer retina contains one rod photoreceptor cell type, adapted to low light (scotopic) vision, and four cone types, mediating color vision at brighter (photopic) illumination. These photoreceptors are arranged in a distinct stereotyped mosaic pattern that crystalizes during larval stages (Robinson et al. 1993; Fadool 2003). The peak sensitivities of zebrafish cones are 360-361 nm (ultraviolet, short single cone), 407-417 nm (blue, long single cone), 473-480 nm and 501-503 nm for the green/red double cone (Allison et al. 2004; Cameron 2002; Nawrocki et al. 1985; Robinson et al. 1993). Hence the visual spectrum of the zebrafish reaches deeper into the infrared than the human and enables ultraviolet vision. Consistent with their diurnal life style, the zebrafish retina features a high proportion of cones. Vision is nearly exclusively cone driven up to 15 to 20 dpf (Raymond et al. 1995; Saszik et al. 1999). In this respect, the larval zebrafish retina mimics cone dominant macular vision of humans.

Horizontal cells of the inner retina receive input from and feed back to photoreceptors, contributing to the center-surround system that enhances contrast sensitivity. These interneurons can be classified into 4 groups ( $\mathrm{H} 1$ to $\mathrm{H} 4)$ which differ in their connectivity to cones and in their gene expression profiles (Klaassen et al. 2016). Bipolar cells are divided into two physiological types depending on their response to changes in illumination: the ON- and OFF-bipolar cells. They can be further subdivided into at least 17 subtypes (Connaughton et al. 2004; Connaughton 2011). Bipolar cells transmit the signal to ganglion cells, whose synapses are found in the inner plexiform layer together with synapses of amacrine interneurons. Amacrine cells are a diverse group of interneurons that modify the signal transmitted from bipolar to ganglion cells. They differ in size, arborization, size of receptive field and biochemistry resulting in a population of neurons that shape signal transmission in the inner plexiform layer in a subtype-specific way (reviewed by (Masland 
2012)). Novel amacrine cell types are still being discovered, e.g. by transgenic techniques (Glasauer et al. 2016). Nuclei of ganglion cells together with displaced amacrine cell bodies, build the innermost ganglion cell layer. Ganglion cells are the output neurons of the retina. Their long axons connect the larval retina with 10 distinct regions of the brain of which the optic tectum is the most prominent (Burrill, Easter, JR 1994).

\section{Retinal development}

In general, zebrafish develop rapidly. However, the development of the retina is particularly precocious. By 60 hours post fertilization (hpf) all retinal layers are morphologically distinct and the vast majority of cells are post-mitotic. Following neurulation, movements of a diencephalic cell population result in two evaginations that form the optic bulbs (Schmitt, Dowling 1994). Later, an invagination in the eye primordia cell mass results in the formation of the optic cup at $24 \mathrm{hpf}$ (Schmitt, Dowling 1994). Cell division taking place at the innermost layer of the optic cup will give rise to retinal cells. All retinal neurons as well as Müller glia cells are descendants from one common multipotent progenitor (Turner, Cepko 1987). Ganglion cells in a ventral patch close to the choroid fissure are the first retinal neurons to exit the cell cycle and start differentiation at around $28 \mathrm{hpf}(\mathrm{Hu}$, Easter 1999). All retinal neurons develop first in a ventro-nasal region and then distribute to more dorsal regions (Kljavin 1987; Schmitt, Dowling 1994). The formation of an inchoate ganglion cell layer at around $36 \mathrm{hpf}$ is followed by differentiation of neurons located in the INL (amacrine and horizontal cells at $50 \mathrm{hpf}$ followed by bipolar cells at $60 \mathrm{hpf}$ ). Photoreceptors become postmitotic starting from $43 \mathrm{hpf}$ while the photoreceptor layer can be observed histologically from 48 hpf on (Schmitt, Dowling 1994; Branchek, Bremiller 1984). Müller cell markers HNK-1 and glutamine synthetase are first detected by $60 \mathrm{hpf}$ (Peterson et al. 2001). 
However, Müller cell maturation seems to underlie a biphasic pattern, as other typical markers for these cells are only found at later stages (Peterson et al. 2001). Once photoreceptors exit the cycle, they start to mature and express opsins. Paradoxically, rod photoreceptors that become functionally integrated last are the first to express rod opsin, followed by red, green, blue and later by UV cones (Raymond et al. 1995; Saszik et al. 1999). Coinciding with the development of photoreceptor outer segments and formation of ribbon synapses with second order neurons at around $60 \mathrm{hpf}$, the first behavioral responses to light can be observed (Branchek, Bremiller 1984; Easter, Nicola 1996; Biehlmaier et al. 2003). Signal transmission from photoreceptors to bipolar cells sets on around $3.5 \mathrm{dpf}$, coinciding with the time when first electroretinogram (ERG) responses can be recorded (Branchek 1984).

In a sense retinal development is never finished, since the retina continues to grow in adulthood by adding cells in the ciliary margin of the retina (Wan et al. 2016). The retina also has immense regenerative properties that are increasingly investigated. Müller glia cells can dedifferentiate and replenish all retinal cell types after damage (reviewed by (Goldman 2014).

\section{Retina function}

Given the rapid development of the zebrafish retina, it comes as no surprise that retinal function is already apparent at larval stages and can be assessed by both electrophysiological and behavior means.

The most direct electrophysiological approach is the recording of electroretinograms (ERG), which records the outer retina response to light. For this purpose the electrode is placed on the cornea, recording sum-field potentials in response to light (Makhankov et al. 2004). Practically both intact larvae and eye cup preparations are suitable for such measurements. 
The first ERG responses in the zebrafish can be recorded starting from about $3.5 \mathrm{dpf}$, yielding robust cone-mediated response from $5 \mathrm{dpf}$ on (Brockerhoff et al. 1997; Seeliger et al. 2002; Makhankov et al. 2004; Saszik et al. 1999). Functional rod input starts between 15 and 21 dpf (Bilotta et al. 2001; Branchek 1984; Saszik et al. 1999). Zebrafish display canonical vertebrate ERG responses consisting of a negative a-wave reflecting hyperpolarization of photoreceptors, followed by a positive b-wave reflecting activity of ONbipolar cells. Furthermore, one can readily assess the OFF-response (d-wave), reflecting OFF bipolar activity. The most common recordings consist of a series of white light stimuli of increasing light intensities to assess response thresholds and response amplitudes. Furthermore the flicker ERG can be used to determine the flicker-fusion-frequency, where single responses to a train of flashes cannot be resolved anymore (Branchek 1984). Spectral ERG allows to investigate the function of single cone subtypes by application of monochromatic light stimuli (Hughes et al. 1998), which can especially be advantageous to probe UV-sensitive cones as they contribute very little to the normal white light ERG (Zang et al. 2015). Defects in ganglion cells do not influence the ERG response (Gnuegge et al. 2001). One way to study ganglion cell function is extracellular recordings of the optic nerve on isolated eyes, a method that can be combined with simultaneous ERG recordings (Emran et al. 2007; Li, Dowling 2000). More sophisticated electrophysiological measurements have been used to measure whole cell currents of ganglion cells (Gnuegge et al. 2001; Zhang et al. 2010). Physiological properties of the remaining retinal neurons has been assessed by patch-clamp recordings or suction-electrode recordings for photoreceptors in isolated retinas or isolated cells (Enright et al. 2015; Vroman et al. 2014; Brockerhoff et al. 2003). However, the limited number of publications on recordings in retinal slices shows one of the limitations of the zebrafish model to study retinal physiology (Connaughton et al. 2008). 
Especially at larval stages, the small zebrafish retina makes cellular electrophysiology challenging. However, development of different optical biosensors that visualize $\mathrm{Ca}^{2+}$ or $\mathrm{Cl}^{-}$ concentrations or membrane voltages can, at least partially, compensate for the challenging cellular electrophysiology (see below).

The other functional approach to visual system function testing is behavioral assays that comprehensively test visual processing of the entire visual system in a non-invasive manner. Zebrafish primarily use vision for prey hunting and predator avoidance, necessitating a quick emergence of visual guided behavior. The startle response is the first visual guided behavior developing at around $68 \mathrm{hpf}$ (Easter, Nicola 1996), likely mimicking an escape response from a looming predator (Kimmel et al. 1974). While the startle response is not based on form vision, the optokinetic response (OKR), also seen from day 3 on represents the first visual guided behavior requiring form vision (Clark 1981). The OKR is a robust behavior of the fish eyes tracking the moving surround. For experimental purposes, the zebrafish larva is typically immobilized in a viscous solution surrounded by a paper drum that presents rotating black and white bars. The elicited behavior consists of a smooth pursuit movement in the direction of the presented stimulus and a fast saccadic resetting movement. Given the large size and dark pigmentation of the eyes, tracking of the eye movements enables quantitative assessment of the visual performance. Computer based OKR setups allow more sophisticated measurements, e.g. presentation of stripes of different intensities or different rotation velocities assessing contrast vision and temporal resolution respectively (HuberReggi et al. 2013; Rinner et al. 2005). Since this behavior is largely reflexive it has been successfully used to screen for blind mutant strains (Brockerhoff et al. 1995; Neuhauss et al. 1999; Muto et al. 2005). An alternative tool to unravel blinding disorders is the visual motor 
response (VMR). For VMR tests the locomotor activity upon illumination changes can be tracked during several hours with alternating periods of darkness and light. Stereotypic increase and decrease in locomotor activity can be observed at light offset and onset respectively (Emran et al. 2008). Another powerful tool, also suitable for large-scale examination of visual impairment, is the optomotor response (OMR). To evoke an OMR, a screen displaying moving black and white bars is presented to zebrafish from below or the side of the tank. Zebrafish at age of $7 \mathrm{dpf}$ or older will respond by swimming in the direction of the moving bars, either escaping a dark or following a white stripe (Neuhauss et al. 1999). Schooling behavior in adult zebrafish influences the OMR, hampering large scale screens at later stages. The adult escape response is another behavioral assay that allows probing for visual impairment in adult zebrafish. The fish is placed in a round tank that is surrounded by a white paper drum with one black stripe that is mimicking a predator and contains a pole in the center. Zebrafish with intact vision exhibit a robust escape response from the black bar and try to hide behind the central pole (Li, Dowling 1997).

The possibility to resolve retina development and morphology at cellular resolution with ready functional read outs make the retina an ideal system to study neural circuit development and its link to behavior. Similarly mutant strains with visual impairment can readily be identified (reviewed in (Gestri et al. 2012; Malicki et al. 2016)).

\section{TRANSGENESIS BASED APPROACHES}

Transgenesis, the introduction of an exogenous piece of DNA into the genome of the host organism, is a powerful technique to label, ablate, and monitor cells in a living animal. This approach is particularly fruitful in the zebrafish with its transparent embryos and rapid development. The optical clarity of zebrafish embryos and larvae allow sophisticated live 
imaging to directly follow cell movements during development, fate map developmental lineages, ablate specific cells, and study neuronal activity related fluorescent changes. A number of transgenic approaches have been applied in the past, while the adaptation of the host factor independent Tol2 transposon system, originally discovered in the Medaka, has greatly facilitated transgenesis in zebrafish (Kawakami et al. 2000).

Application of Tol2-mediated transgenesis to study retinal development and function is booming, in no small part due to the ever increasing number of marker genes (usually GFP (green fluorescent protein) derivatives) and activity markers (GCaMP variants).

\section{Transposon based transgenesis}

A number of transposon system work in the zebrafish, such as the salmonid fish sleeping beauty (Balciunas et al. 2004), Caenorhabditis elegans Tc3 (of the Tc-1/mariner family) (Raz et al. 1998; Fadool et al. 1998), Medaka Tol2 and Tol1 (Koga et al. 2008; Kawakami et al. 2000) and the maize $A c / D s$ transposable elements (of the hAT family) (Quach, Helen Ngoc Bao et al. 2015). The efficiency of Tol2-mediated transgenesis, permitting insertion of genetic material of more than $100 \mathrm{~kb}$ into the zebrafish genome, have now replaced most other transgenic systems (Suster et al. 2011).

The Tol2 injection mix consisting of transposase-encoding mRNA and a transposon donor plasmid containing a Tol2 flanked reporter gene of interest fused to cell type specific regulatory elements. This mix is injected into the one cell stage zebrafish embryo, enabling transposase mediated excision of the Tol 2 construct from the donor plasmid and integration into the genome (Figure 2a) (Kawakami 2007). Cells will experience random integration at multiple sites at different times during early embryogenesis resulting in a genetically mosaic 
animal. This applies also to the germ line necessitating inbreeding at the next generation to yield stable transgenic lines (Kawakami 2007).

The randomness of integration may in few cases lead to the disruption of endogenous genes or regulatory elements, which can be exploited to induce mutations (see section on Insertional Mutagenesis).

The Gateway cloning based Tol2kit, greatly facilitates generation of Tol2 donor plasmids where 3 entry vectors are converted to a destination vector by att site specific recombinational cloning (Kwan et al. 2007). This kit has greatly advanced Tol2 mediated transgenesis, allowing selection of an expanding variety of existing clones (http://tol2kit.genetics.utah.edu/index.php/Main_Page).

Random integration of the Tol 2 construct at multiple insertion sites can be circumvented by using phic31-mediated site directed transgenesis. This approach is based on phiC31 integrase-mediated single insertion of a transgene vector into attP (attachment site Phage) landing sites (Mosimann et al. 2013; Hu et al. 2011; Roberts et al. 2014). A number of lines with attP landing site at defined genomic locations have been generated. Injection of phiC31 integrase mRNA together with an attB (attachment site Bacterium) site containing donor plasmid allows single integration of the cassette at the attP landing site by recombination with high efficiency in both somatic and germ cells (Figure 2b) (Mosimann et al. 2013; Roberts et al. 2014). Germline transmission rate was reported to reach values between $10 \%$ (Roberts et al. 2014) and 34\% (Mosimann et al. 2013). Integration events create attR and attL (right and left) sites which are incompatible for phiC31 mediated recombination thus irreversible transgene integration is ensured (Groth et al. 2000). attP flanked reporter genes can be used to establish transgenic lines via recombinase-mediated cassette exchange, 
allowing generation of a single-insertion transgenic line within one generation (Mosimann et al. 2013; Roberts et al. 2014; Hu et al. 2011). This single integration approach reduces variability due to position effects and multiple insertions. The future will likely see more use of this approach with the availability of more transgenic lines with defined landing sites.

\section{Two-component systems}

An important advance of the described transgenic technology is the use of binary systems, pioneered in Drosophila and the mouse. These systems allow additional flexibility and ease of generating cell specific expressing strains. They allow crossing driver lines with various reporter or switch lines generating new transgenic lines purely by crossing existing strains.

One widely used system, originally established in Drosophila, is the Gal4/Upstream Activating Sequence (UAS) system (Figure 2c). Gal4 is a yeast transcriptional activator that specifically recognizes the UAS sequence and drives expression of any ORF immediately downstream of it (Guarente et al. 1982). The two-component system consists of a Gal4 driver line that expresses Gal4 under the control of a tissue specific regulatory region and a UAS reporter line harboring a cassette of UAS sequence and a downstream open reading frame (ORF). By crossing the driver with the reporter line, the ORF is expressed in all cells that have promoter activity (Figure 2c). In order to obtain stronger Gal4 activity a number of modified Gal4 variants have been generated. One commonly used variant is Gal4-VP16 which possesses the transcriptional activation domain of the herpes simplex virus VP16 (Sadowski et al. 1988) and variants thereof, such as the weaker Gal4FF (Asakawa et al. 2008). One drawback of the Gal4/UAS system is that UAS transgenes are prone to show position effects which may result in gene silencing or variegated transgene expression levels 
(Asakawa, Kawakami 2008). This limitation may be overcome in the near future by using the PhiC31 system.

The Cre/Lox system, pioneered in rodents, is another popular two component system, which is especially suitable for lineage-tracing, connectome analyses and conditional gainand loss-of-function studies. It is based on the bacteriophage P1 Cre recombinase, which catalyzes recombination between locus of X-ing over (lox) sites (Sauer 1987). For spatial control of transgenesis, the Cre driver line expresses Cre recombinase under the control of a specific promoter $\mathrm{X}$. This line is then crossed with switch transgenic line that harbors a cassette of a promoter $Y$ upstream of a lox site flanked (floxed) ORF or a stop cassette followed by a second cargo ORF. Lox sites can be in tandem, wherein Cre-mediated recombination results in circularization and excision of the cassette or in a head-to-head orientation that promotes inversion of the floxed cassette (Branda, Dymecki 2004). In cells where promoter $\mathrm{X}$ is active, Cre recombines lox sites leading to excision of the floxed ORF or stop cassette ceasing its expression and thus allowing promoter $Y$ to drive expression of the downstream cargo (Figure 2d).

Multicolor labelling of cells mediated by Cre mediated recombination of a cassette of three different floxed fluorescent proteins (Brainbow, see below) provides a prime example for the application of the Cre/Lox system in lineage analysis or connectome analyses (Pan et al. 2011).

\section{Temporally controlled inducible transgenesis}


While spatially controlled transgenesis can be achieved by cell type specific promoters and the use of the described two-component systems, additional temporal control by exogenous induction is often desirable. A number of systems have been established in zebrafish.

Temporal control over transgene expression can be achieved by using heat shock promoters (the hsp70 promoter is commonly used) (Halloran et al. 2000). Taking Cre recombinase under heat shock control enables both spatial and temporal control of gene expression (Thummel et al. 2005; Le et al. 2007). This method is somewhat limited by the observed leakiness of the hsp70 promoter, resulting in basal activation of Cre at permissive temperatures and hence non-conditional recombination (Hans et al. 2009).

Another way to accomplish temporal control of recombination is provided by the CreER ${ }^{\mathrm{T} 2}$ system wherein Cre recombinase is fused to a mutated version of the human estrogen receptor (ER ${ }^{\top 2}$ ) (Feil et al. 1997). Administration of the estrogen derivatives Tamoxifen or 4$\mathrm{OHT}$ results in activation of $\mathrm{CreER}^{\mathrm{T} 2}$-mediated recombination by induction of conformational changes in the ER ligand-binding domain (Feil et al. 1997). This leads to shedding of endogenous Hsp90 protein and translocation of $\mathrm{CreER}^{\mathrm{T2}}$ to the nucleus, where recombination is catalyzed (Hans et al. 2009). Fusing mCherry and CreER ${ }^{T 2}$ in a single ORF separated by a viral T2A peptide sequence results in production of mCherry and CreER ${ }^{\mathrm{T} 2}$ in a stoichiometric ratio, which allows visual screening of low-expressing $\mathrm{CreER}^{\mathrm{T} 2}$ driver lines thus decreasing probability of non-conditional Cre activity (Hans et al. 2011).

Another system for spatial and temporal control over gene expression is achieved by the Tet-ON binary system. This system consists of a transcription factor, the reverse tetracycline-controlled transcriptional activator (rtTA) and the tetracycline response element (TRE) upstream of the gene to be transgenically expressed (Gossen, Bujard 1992; 
Gossen et al. 1995). Spatial control over gene expression is achieved by placing the transgene cassette (containing rtTA and TRE) under the control of a tissue-specific promoter (Huang et al. 2005). Gene expression can now be controlled by the addition of tetracycline or doxycycline which both induces expression of rtTA (Gossen, Bujard 1992; Gossen et al. 1995). Without application of tetracycline or doxycycline, the gene downstream of TRE is not expressed. Due to leakiness in transgene expression in zebrafish, the system had to be improved by fusion of the rtTA to a mutated glucocorticoid receptor or a domain of the ecdysone receptor that resulted in abolishment of the leakiness and also made the system reversible (Knopf et al. 2010). Employing this technique, two zebrafish retina Tet-ON driver lines were generated, one driving rtTA expression specifically in UV cones and the other one in rods (West et al. 2014; Campbell et al. 2012).

\section{Application of transgenic approaches}

The presented transgenic technology hands the retinal researcher a large variety of tools to study all aspects of retinal development and function. Recent advances in protein engineering of stable fluorescent proteins, photo-convertible fluorophores and dyes that allow neuronal activity monitoring complement this tool box. The parallel development of advanced microscopic techniques, such as multi-photon, selective planar illumination (SPIM) and super resolution microscopy unlocks the full power of these approaches.

\section{Gene overexpression}

Overexpression assays provide (additionally to loss-of-function assays) a tool for gene function analysis. In such a gain-of-function experiment, a gene of interest is overexpressed and subsequent phenotype analysis is performed. Gene overexpression can be achieved in 
two ways: injection of expression constructs in form of DNA or mRNA. mRNA injection has the advantage that it leads to uniform expression over the whole embryo, while DNA leads to mosaic expression (Malicki et al. 2002). Nonetheless, mRNA gets degraded over time and dilutes with cell divisions.

One application in retinal research is the generation of a disease model by overexpressing a gene of choice containing the diseases causing mutation. For instance the expression of a mutated form of retinal guanylate cyclase lead to altered retinal morphology providing a model for the corresponding human cone-rod dystrophy (Collery et al. 2013).

\section{Cell labeling and fate mapping}

Labelling of cells with fluorescent reporters has greatly contributed to our understanding of cell-cell interactions, cell proliferation, cell migration and the embryonic origins of tissues. This area of retinal research is probably the most advanced with numerous studies describing the development of cell types and their connections.

Transgenic lines labeling specific cell types can be used to follow this cell type throughout development. A beautiful example is the Spectrum of Fates technique that allows the monitoring of all retinal cell types simultaneous during development (Almeida et al. 2014).

Connectome studies are facilitated by using transgenic lines with fluorescently tagged cell types. For instance the connection between photoreceptors and second order neurons (bipolar cells and horizontal cells) has been described in detail using fluorescent transgenics that label all photoreceptor subtypes in combination with lipophilic dyes to mark bipolar or horizontal cells ( $\mathrm{Li}$ et al. 2012; Li et al. 2009). These studies revealed that red cones are exclusively contacted by $\mathrm{H} 1$ horizontal cells, while blue, green and UV cones are contacted 
by two or even all three cone horizontal cell subtypes ( $\mathrm{H} 1$ to $\mathrm{H3}$ ) (Li et al. 2009). Furthermore, transgenic labelling of photoreceptors has nicely contributed to the our understanding how the stereotyped crystalline photoreceptor mosaic emerges during development (Allison et al. 2010; Fadool 2003).

In the inner retina, the trajectory of ganglion cells has been studied in detail in transgenic larvae with red labelled RGCs (expressing mCherry) and green synapses (EGFP-tagged synaptic protein synaptophysin) which allowed analysis of the RGC connectome and the celltype specific projection pattern (Robles et al. 2014).

Finally transgenic technology allowed the in vivo imaging of cell division and migration of newborn cells in the retina. Lineage tracing experiments using transgenics demonstrated that cone precursor cells in the ciliary marginal zone $(\mathrm{CMZ})$ are dedicated to produce a single type of cone. Hence each of the four cone subtypes has its own dedicated precursor (Suzuki et al. 2013).

Transgenic labeling can also lead to the identification of previously unknown cell types. For instance a transgenic line expressing GFP under the control of the mGluR6b promoter identified a novel cholinergic, non-GABAergic, non-starburst amacrine cell type (Glasauer et al. 2016).

One current limitation of these labelling approaches is the paucity of well characterized regulatory elements that drive cell type specific expression. Furthermore, labelling of a dense population of cells often prevents tracing trajectories of single cells. This problem can either be solved by mosaic expression of a transgene or by the multicolor brainbow system.

Brainbow is a Cre/lox based transgenic approach consisting of a promoter that controls expression of a cassette of three different fluorescent reporters, RFP, CFP and YFP (Gupta, 
Poss 2012; Pan et al. 2011; Pan et al. 2013). Mutually incompatible lox site pairs are used to yield a stochastic choice of expression of only one of the three fluorescent proteins per copy of construct (Pan et al. 2011). Different lines have been generated, with only single insertions leading to expression of 3 different colors in heterozygotes and 6 colors in homozygotes or multi insertion animals that express a wide range of different hues (Pan et al. 2013). Furthermore by using different promoters or combining the system with the Gal4/UAS system, tissue specific labelling can be achieved. Work in mosaic animals allows sparse labelling of cells facilitating tracking of trajectories. In a recently published work, authors availed themselves of the brainbow system ( $\operatorname{Tg}($ UAS:Zebrabow $))$ to characterize three different clones of retinal stem cells within the CMZ. They showed the CMZ to consist of dormant CMZ tip cells (Type I), a Type II clone of proliferating cells and a Type III clone giving rise of differentiating clones (Tang et al. 2017).

Another useful technique is the use of photoconvertible fluorescent proteins, such as Kaede, Dendra2, and Dronpa. Both, Kaede and Dendra2 irreversibly photoconvert when activated with UV-light of $400 \mathrm{~nm}$ (Kaede) or light of $488 \mathrm{~nm}$ (Dendra2) (Ando et al. 2002; Wachter et al. 2010). Dronpa on the other hand is a GFP-like fluorophore and can be reversibly switched from dim irradiance to a bright state with $405 \mathrm{~nm}$ light. The bright state can be switched off to the dim state by $488 \mathrm{~nm}$ light irradiation (Habuchi et al. 2005). By either switching on fluorescence (Dronpa) or switching color (Kaede and Dendra2) one can visualize cells in a background of non-labelled or differentially labelled cells, facilitating fate-mapping and observation of cell-division and migration. An application of this approach led to a description how variable clones can give rise to a invariant retina, a discovery of importance not only for retinogenesis (He et al. 2012). 


\section{Cell ablations}

Transgenic tools can be used to ablated specific cell, e.g. by expressing toxins or laser ablating labeled cells. The Nitroreductase cell ablation method has been popular in the zebrafish, since it allows spatial control via transgene expression and temporal control by prodrug addition to the water. The bacterial enzyme Nitroreductase (NTR) converts the prodrug Metronidazole (Mtz) into a cytotoxic agent that causes DNA damage and subsequent cell death of NTR expressing cells (Curado et al. 2007).

This method was used to selective ablate a specific subset of bipolar cells, originally identified in a Gal4 based gene trap approach (Zhao et al. 2009).

Such ablation studies are particularly important to further our knowledge on regeneration studies. Ablated cells of the retina are usually replenished by stem cells origination from dedifferentiated Müller glia (reviewed by (Goldman 2014)).

Rod photoreceptors can be replenished by either dedifferentiated Müller glia cells or by rod precursor cells that reside in the ONL. One study using the aforementioned cell ablation technique could show that the extent of rod loss predicts which precursor pool is used. When only a subset of rod cells are ablated the rod precursor pool is used to replenish them. Conversely, the regenerative response of Müller glia cells is trigger by large scale and rapid loss of rods (Montgomery et al. 2010).

Another question that can be asked with current technology is if regenerated neurons reconnect with the synaptic partner of the originally ablated cell. By ablation of a distinct subset of bipolar cells ( $x f z 43), D^{\prime}$ Orazi and colleagues showed that the regenerated neurons do not reconnect with the exact same cells as the ablate cells. Hence regenerated neurons employ a distinct synaptic matching strategy (D'Orazi et al. 2016). 


\section{Neuronal Activity Monitoring}

Traditionally, electrical activity of neurons is measured by electrophysiology using either sharp electrode, patch clamp or extracellular electrode recording techniques. These technically challenging experiments achieve insight into the function of single (or small groups of) neurons at high temporal resolution. The parallel measurements of many neurons by multi-electrode arrays are impossible for most many neural circuits and necessitate ex-vivo preparations.

Recent developments in protein engineering have led to the establishment of genetically encoded sensors of neuronal activity, such as $\mathrm{Ca}^{2+}$ indicators that can partially compensate for the challenging electrophysiology, even though temporal resolution is not comparable to electrophysiology. Importantly, functional neuronal imaging enables the concomitant analysis of potentially thousands of cells, potentially covering the complete neural network. This approach is particularly well suited for the small and transparent zebrafish brain, which more than compensates for the difficulties to perform cellular physiology in the small larva.

Intracellular calcium concentration is a good proxy for a neuron's excitable state. Monitoring $\mathrm{Ca}^{2+}$ dynamics in neurons using genetically encoded calcium indicators (GECls) is therefore a powerful tool to visualize not only general neuronal activity, but also $\mathrm{Ca}^{2+}$ homeostasis in healthy and diseased neurons. Synaptic $\mathrm{Ca}^{2+}$ dynamics are crucial for synaptic vesicle release and thus signal propagation. A great advantage of neuronal activity monitoring by $\mathrm{Ca}^{2+}$ imaging is that it can be performed non-invasively in living larvae and can even be combined with consequent behavioral output. A variety of GECls have been engineered. The two most commonly used GECls are FRET (Förster Resonance Energy 
Transfer) based sensors and GCaMP calcium indicators. The later ones are the most popular ones used in zebrafish.

GCaMPs are fusion proteins consisting of a circularly permuted EGFP (enhanced GFP) which is linked at the N-terminus to the M13 peptide of the myosin light chain kinase and on the Cterminus to Calmodulin (Nakai et al. 2001). When $\mathrm{Ca}^{2+}$ binds Calmodulin, Calmodulin interacts with its target sequence M13 which induces a conformational change in EGFP that results in enhanced fluorescence (Nakai et al. 2001). These sensors are constantly improved in terms of their sensitivity and kinetics (reviewed by (Broussard et al. 2014)).

In comparison to GCamps, FRET sensors like Cameleon are dual fluorophore based. Binding of $\mathrm{Ca}^{2+}$ to the Calmodulin of Cameleon leads to a Calmodulin-M13 interaction and resulting conformational changes allow fluorescent resonant energy transfer from one fluorophore to another one (Miyawaki et al. 1999). This results in a change of the emitting color (Miyawaki et al. 1999).

Synaptic activity can be monitored using the genetically encoded reporter SyGCamp, a GECI that localizes to the synapse. During neuronal activity, $\mathrm{Ca}^{2+}$ entering the synapse through voltage-gated $\mathrm{Ca}^{2+}$ channels induce the release of neurotransmitter filled vesicles into the synaptic cleft. SyGCamp consists of a GCamp fused to the synaptic protein synaptophysin. In vivo $\mathrm{Ca}^{2+}$ imaging using encoded SyGCamps enables monitoring the brief presynaptic $\mathrm{Ca}^{2+}$ transient thus allowing detection of synapse activation (Dreosti et al. 2009). This approach is especially suitable for monitoring neuronal activity in the retina, since retinal neurons do not transmit information in an all-or-none action potential fashion but rather by graded voltage changes. Hence in contrast to most central nervous system neurons, where action potential firing neurons can be images as nicely "blinking" neurons, retinal neurons only show subtle changes in fluorescent intensity (Dreosti et al. 2009). 
Hence $\mathrm{Ca}^{2+}$ imaging on retinal neurons in zebrafish have greatly advanced the understanding on synaptic function in the retina, such as how amacrine cells modulate synaptic output of bipolar cells (Rosa et al. 2016) or how the volume of bipolar cell terminals influences signal transmission (Baden et al. 2014).

$\mathrm{Ca}^{2+}$ imaging experiments can further be employed to monitor degenerating cells and have led to the insight that increased cytoplasmic $\mathrm{Ca}^{2+}$ might not be the underlying cause for photoreceptor degeneration in a phosphodiesterase (pde6c) mutant fish (Ma et al. 2013).

One drawback of $\mathrm{Ca}^{2+}$ imaging is the limitation in the field of view. One might miss interesting events, simply because they are outside of the region being imaged. A solution to that is the so called Campari method. It allows to permanently labelling active neurons in a timely controlled manner. Campari has a similar structure as GCamps, but instead of a circularly permuted EGFP, EosFP is used as the fluorescent protein, which is bright green and converts to red emission upon exposure to violet light (Fosque et al. 2015). Active neurons are labelled by a green-to-red conversion only under violet illumination, allowing precise timing of activity-mapping.

While GECls are widely used in zebrafish to monitor visual processing its use in the retina is restricted by the prevalence of non-spiking neurons.

Hence the advance of imaging methods to monitor extracellular glutamate, the sole neurotransmitter of photoreceptors, constitutes an important advance for zebrafish retinal research. Since photoreceptors and bipolar cells modulate the tonic release rate of glutamate in response to graded changes in membrane potential via specialized ribbon synapses, synaptic glutamate concentrations are a good proxy for neuronal activity.

iGluSnFr is a glutamate biosensor consisting of circularly permutated GFP and Gltl, a bacterial glutamate transporter (Marvin et al. 2013). Glutamate transients lead to binding of 
glutamate to iGluSnFr and induce a conformational change evoking an increase in fluorescence emission. Fast kinetics, its robust specificity to glutamate and high signal-tonoise ratio make it a powerful tool to monitor neuronal activity (Marvin et al. 2013). Glutamate dynamics in the zebrafish retina can now be directly observed using the recently introduced transgenic line $\operatorname{Tg}(g f a p: i G l u S n F R)$, that expresses the glutamate sensor in Müller glia cells (MacDonald et al. 2016).

A number of additional optic sensor are currently developed that may benefit zebrafish retinal research in the future. For instance an optical synaptic $\mathrm{pH}$ sensor was used to study the still elusive feedback mechanism of horizontal cells to photoreceptors in the zebrafish retina (Wang et al. 2014).

\section{MUTAGENESIS}

The traditional genetic approach to understand gene networks is the removal of gene function followed by inspection of the resulting phenotype. This approach has been pioneered more than 100 years ago and its impact on biology can hardly be overestimated. Historically forward genetic approaches were first used by randomly inducing mutations followed by the description of the resulting phenotypes. More recently the advent of modern molecular genetics enabled researchers to use reverse genetics by first inactivating a known gene of interest before studying the resulting phenotype.

\section{Forward genetics}

The strength of forward genetics is that this approach is completely unbiased and needs no prior knowledge of genes and pathways potentially affecting the biological process of interest. Zebrafish was the first vertebrate model organism where large scale mutagenesis screens aiming at saturating mutagenesis was attempted. 


\section{Chemical mutagenesis}

Inspired by successful saturating chemical screens in C. elegans and Drosophila (Hirsh, Vanderslice 1976; Nusslein-Volhard, Wieschaus 1980), two large-scale (Driever et al. 1996; Haffter et al. 1996) as well as several small-scale genetic screens were performed. All these screens are based on chemical mutagenesis approaches using the chemical N-Ethyl-Nnitrosourea (ENU) to induce point mutations and small deletions in the germline of male zebrafish. These mutations were bred to homozygosity in a simple inbreeding scheme (Mullins et al. 1994; Solnica-Krezel et al. 1994). Alternative screening methods based on parthenogenesis were also used to a lesser extent (reviewed by (Patton, Zon 2001)). These screens proved to be very powerful and were basically limited only by the observational capabilities of the screener and the logistics of such a large scale operation. More than a thousand loci have been identified, including many affecting retina morphology and function (Brockerhoff et al. 1995; Malicki et al. 1996; Neuhauss et al. 1999; Muto et al. 2005). Besides locomotion and hearing, the visual system was the first system where behavioral screens have been performed. However, the molecular identification of the responsible loci proved to be a bottleneck, demanding tedious genetic mapping and positional cloning efforts. Exome sequencing only recently became an alternative (Kettleborough et al. 2013). Therefore, even 20 years after the initial description, there are still a number of interesting mutant strains that have not been linked to the underlying genetic defect.

\section{Insertional mutagenesis}

The challenge of positional cloning of mutated genes motivated efforts to achieve insertional mutants where the mutation is caused by the insertion of a DNA element. Such 
an insertion does not only disrupt the affected gene, but also tag it, facilitating subsequent identification.

This was first achieved by utilizing a pseudotyped retrovirus (Amsterdam et al. 1999). These viruses contain a genome based on the Moloney murine leukemia virus and are pseudotyped with the envelope of a vesicular stomatitis virus, allowing them to infect all cells. Virus particles are injected into 1000-2000 cell stage embryos with the aim of targeting primordial germ cells present at this stage (Amsterdam et al. 1999; Gaiano et al. 1996a; Gaiano et al. 1996b). Founder fish are in-crossed to generate F1 families that are tested for inserts by Southern blot analysis. Multi-insert F1 fish are inbred and sibling F2 fish are crossed to allow identification of phenotypes of recessive mutations in the resulting F3 generation (Amsterdam et al. 1999). Although the mutation rate was quite low, the underlying mutated genes could be very efficiently identified. Some retinal mutations and their underlying genetic defect were identified by this route (Gross et al. 2005).

The expertise needed for handling of pseudotyped virus that are difficult to produce in high titers and are a potential biohazard by being infectious for humans, prevented the wide spread use of this technique.

Transposon based insertional mutagenesis became a possibility with the advent of efficient transgenesis. Additionally to gene disruption, insertion of a marker gene (e.g. GFP) readily provides information on the expression pattern of the disrupted gene (Balciunas et al. 2004; Kawakami et al. 2004; Kotani et al. 2006). The mutation rate is low compared to chemical mutagenesis, but the identification of the disrupted locus is straight forward (Nagayoshi et al. 2008; Balciuniene et al. 2013). Due to the low mutagenesis rate only few mutant strains have been identified. 


\section{Reverse genetics}

While forward genetics is unbiased, reverse genetics is hypothesis driven by manipulating a gene with a prior suspected function. This is of particular interest with gene orthologs that have been linked to human diseases, since disease mechanisms can potentially be uncovered in a direct genetic zebrafish model of a genetic disease of interest.

Due to the lack of an efficient reverse genetics mutagenesis tool, antisense mediated gene knockdown was for a long time the only method available and thus extensively used to target a gene of interest. Strictly speaking this is not a reverse genetic technique, since no heritable change is induced. Gene function was downregulated by the injection of morpholino antisense nucleotides at the one cell stage. These antisense nucleotides are modified to make them more stable in a cellular environment and less toxic. The sequences are either designed to block the translational start sites or splice sites. Knockdowns allow rapid assessment of gene function in larvae up to $5 \mathrm{dpf}$ (Nasevicius, Ekker 2000). At later stages no efficient knockdown can be achieved due to dilution by increased cell numbers of the growing fish. Morpholino injections allow for a sometimes deceptively quick analysis of gene function. As in all proper experiments, morpholino effects need to be carefully controlled to avoid premature conclusion on phenotypes that may be caused by toxic effects. This is particularly pertinent for phenotypes involving degeneration (Kok et al. 2015). An additional advantage of morpholinos is that also maternally provided mRNA is targeted, that may mask phenotypic effects at earlier stages in embryonic lethal mutants.

One of the first realized real heritable reverse genetic approach is TILLING (Targeting Induced Local Lesions in Genomes) pioneered by plant geneticists (McCallum et al. 2000; Till et al. 2007; Gilchrist et al. 2006; Winkler et al. 2005). This technique is somewhat placed between forward and reverse genetics in that initially mutants are randomly generated by 
chemical mutagenesis. The originally mutated male fish are outcrossed, essentially generating a swimming library of numerous mutations, with each fish carrying a multitude of heterozygous mutations. By sequencing or by an endonuclease cleaving heteroduplex DNA (Cel1) approach, mutations are identified and recovered by mating the fish carrying mutations in the gene of choice (Wienholds et al. 2003; Draper et al. 2004; Wienholds et al. 2002; Oleykowski et al. 1998). Many mutant libraries have been generated, with the biggest one at the Welcome Trust Sanger Center carrying roughly $36^{\prime} 000$ alleles in more than 730 genes (https://www.sanger.ac.uk/sanger/Zebrafish_Zmpbrowse). Although many mutant lines that are used around the world have been generated in this way, the effort to breed lines containing only the desired mutation and the logistics necessitating dedicated centers are drawbacks of this method. Thus, with the emergence of efficient reverse genetic mutagenesis tools, TILLING based approaches are losing popularity.

Two Fokl based genome editing techniques have been successfully established in zebrafish (Figure 3). Fokl is an endonuclease that induces double strand breaks when dimerized (Bitinaite et al. 1998). Both approaches use fusion proteins that fuse Fokl with specifiable DNA binding domains. These sequences specific domain direct Fokl to the genomic target of choice. If two Fokl proteins are in this way joined at a genomic site, a double strand break is induced in the DNA. The error-prone - repair mechanism (non-homologous end-joining, NHEJ) relegates the two DNA strand frequently introducing small insertions or deletions (indels). The two methods differ by the nature of the DNA targeting protein, suing zinc fingers domains (Figure 3a) or TALE (Transcription-Activator-Like Effectors) domains (Figure 3b) (Meng et al. 2008; Doyon et al. 2008; Moore et al. 2012). Transcription-Activator-Like 
Effector Nucleases (TALENs) proved to be more user-friendly and efficient in inducing targeted mutations (Huang et al. 2012).

Although a number of mutants affecting the development of the retina and the eye (e.g. (Deml et al. 2015; Miesfeld et al. 2015)) have been generated by these methods, the more efficient CRISPR/Cas9 method is quickly replacing them.

Genome editing has been revolutionized not only in the zebrafish with by the application of the CRISPR/Cas system. The clustered regularly interspaced short palindromic repeats (CRISPR) - CRISPR-associated (Cas) system in bacteria and archaea targets and cleaves foreign intruding virus and plasmid DNA (Gasiunas et al. 2012). The type II CRISPR/Cas9 system of the bacterium Streptococcus pyogenes is one of the most extensively studied members of the endonuclease family and has been successfully adapted for directed genome manipulation in a variety of species, where a guide RNA directs target-specific induction of double strand breaks by the Cas9 endonuclease. The active CRISPR/Cas9 holoendonuclease in vivo consists of the endonuclease, transcribed from the cas9 gene and a complex of two small RNAs, the trans-activating CRISPR RNA (tracrRNA) and CRISPR RNA (crRNA). The system was modified to be used as a mutagenesis tool. The two small RNAs are fused to one single guide RNA (sgRNA) mimicking the tracrRNA:crRNA complex (Figure 4a) (Jinek et al. 2012). Target recognition of the CRISPR/Cas9 complex is ensured by specific Watson-Crick base pairing of complementary 20 nucleotides on the sgRNA with the genomic target DNA (Figure 4a). CRISPR/Cas9 system is the only mutagenesis method to date that relies on Watson-Crick base pairing rather than potentially less specific DNA recognition by proteins (Gaj et al. 2013). A NGG protospacer-adjacent motif (PAM) is required by the Cas9 to be the $3^{\prime}$ end of the 23 nucleotide target in order to cleave the DNA (Jinek et al. 2012). 
Binding of the sgRNA to the genomic target induces endonuclease activity of Cas 9 and leads to DNA cleavage.

In theory, any sequence of 23 nucleotides harboring a 3' NGG PAM can serve as a target. If using T7 RNA polymerase for in vitro transcription of the sgRNA, the sgRNA must start with a 5' GG. Together with the restriction of a 3' NGG PAM, such targets (GG-N18-NGG) are found in the zebrafish exome one every 128 nucleotides (Hwang et al. 2013). However, methods to increase the target range have been suggested, like mismatching the two $5^{\prime}$ nucleotides to GG (GG-N18-NGG), adding additional GG at the 5' end (GG-N20-NGG) or using SP6 RNA polymerase (5'-G(A/G)) (Hwang et al. 2013; Gagnon et al. 2014). Also, enzymes with different targeting requirements are now being increasingly engineered. Target selection is facilitated by several online tools based on empirical testing that predict possible guides (e.g. (Moreno-Mateos et al. 2015; Montague et al. 2014)). It is recommended to either target the beginning of a gene, where a potentially resulting truncated protein in the mutant lacks most functional domains or directly target regions coding for functional domains. These restrictions, besides the required PAM, the recommended high G/C content further decreases number of possible target sites and can be limiting if targeting smaller genes.

sgRNAs can either be injected into the one cell stage embryo together with nuclear localizing Cas9 encoding mRNA or with Cas9 protein. Successful mutagenesis results in induction of double-strand breaks by the Cas9 endonuclease at the target site. Error-prone NHEJ refuses the two free ends often introducing small insertions or deletions (Figure 4a) (Thyme, Schier 2016; He et al. 2015). The CRISPR/Cas9 system is able to induce somatic mutations at very high frequencies (up to $100 \%$ ) and also germline-transmission is very 
efficient (Burger et al. 2016). CRISPR injected fish (FO fish) are highly mosaic, but when outcrossed to wildtype fish, the sibling progeny (F1 generation) shows limited mutation complexity, suggesting that Cas 9 is active in the first 4 hours, when only 4 germline progenitor cells are present (Jao et al. 2013). Two different breeding schemes (Figure 4b) are used to create stable homozygous mutant lines: Outcross of F0 fish to wildtype fish, resulting in first homozygous mutants in the F2 generation or a direct F0 incross, resulting in transheterozygous F1.

Counterintuitively, the main workload for generating CRISPR mutants does not fall on target selection or sgRNA /Cas9 mRNA synthesis, but rather on genotyping. The targeted region has to be PCR amplified and sequencing following cloning is required to detect animals harboring frame-shift mutations. Injecting two sgRNAs in parallel may decrease the genotyping efforts, as often the genomic region between the targets gets excised resulting in deletions that can be detected by gel-electrophoresis, making cloning and sequencing redundant.

The high efficiency of the CRISPR/Cas9 system can induce such a high load of somatic mutations that embryonic lethality may occur. This can be prevented by fusing the cas 9 open reading frame to the $3^{\prime}$ untranslated region of nanos 1 to target Cas9 to the germline, thereby avoiding somatic mutations. This approach eases the generation of mutants with embryonic lethal mutations (Moreno-Mateos et al. 2015).

The CRISPR/Cas9 system has been further tweaked to induce mutations in a spatially or temporally controlled fashion by transgenic expression of cas9 and sgRNA. Two approaches have been successfully implemented, both relying on Tol2 transposon based insertion of constructs. One relies on a one vector system that contains a cassette containing the tissue 
specific promoter upstream of cas9 and the zebrafish specific U6 promoter upstream of a sgRNA (Ablain et al. 2015). The second approach is based on a two vector system where one cassette containing cas 9 under the control of a tissue-specific promoter is injected into a fish that later will be crossed with another fish that was injected with a construct containing one or several specific sgRNAs under the control of a U6 promoter (Yin et al. 2015). Both studies proved that transgenic expression of cas 9 and sgRNA is sufficient to induce somatic biallelic mutations that result in a phenotype, despite highly varying mutation rate and phenotype severity between siblings (Yin et al. 2015).

Tissue specific mutagenesis was also achieved by ubiquitous transgenic expression of cas 9 and injection of sgRNA into a specific tissue followed by electroporation. Yin et al. showed that targeting ascl1a, a gene involved in retinal regeneration by Müller glia cell dedifferentiation and proliferation, resulted in decreased regeneration measured by the number of proliferating cells after light-induced photoreceptor degeneration (Yin et al. 2015).

Temporal control of mutagenesis can be achieved by temporal control of Cas9 expression, e.g. by a heat-shock promoter (hsp). Heat-shock induced tyrosinase inactivation led to hypopigmentation in the eye, however of different extents (Yin et al. 2015).

Although no successful application of these tissue specific mutagenesis regimes have been reported for studies of the retina, they hold great promise for the future.

In comparison to overexpression assays, where a DNA sequence is expressed in addition to endogenous gene expression, targeted knock-ins have the advantage to simultaneously disrupt an endogenous locus while introducing an ORF of interest. This is of particular 
importance for modeling human retinal diseases, where the exact nucleotide change found in a human mutation could be introduced to the fish. While targeted knock-in based on CRISPR/Cas9 is widely and successfully used in cultured cells, its application in zebrafish is only emerging and technical refinements are required in order to achieve efficient in-frame knock-ins. Depending on the experimental approach, a variety of genetic material, reaching from fluorescent reporter genes, mutated genes, stop codon cassettes to antibody recognition tags can be inserted (Auer et al. 2014; Armstrong et al. 2016; Hruscha et al. 2013; Gagnon et al. 2014). So far, genetic material to be integrated was of different length and kind (plasmid versus oligonucleotides) and contained homology arms of different lengths reaching from only a few nucleotides to several hundred bps (reviewed by (Albadri et al. 2017)). The different strategies resulted in successful integration of the donor DNA, however at rather low efficiencies and with high frequencies of out-of-frame integrations or additional indel mutations (Auer et al. 2014; Armstrong et al. 2016; Hruscha et al. 2013), reviewed by (Albadri et al. 2017). At the current pace of innovation it is to be expected that in the near future efficient ways to introduce precise knock-ins will become available in the zebrafish. This would allow retina researcher to recreate heritable retinal disease of humans with single nucleotide precision in the zebrafish.

\section{OUTLOOK}

The retina as an accessible part of the brain has always fascinated neuroscientist by providing compact neural circuits with a defined function. The highly visual zebrafish is an ideal model to advance our understanding of retinal development and function by providing a compact cone dominant retina with numerous genetic and imaging approaches available. In this review we have sketched some of the latest technological development that will 
strongly influence retinal research. While many these techniques are rapidly and constantly improved, their full impact for our understanding of the vertebrate retina has not been fully realized yet. We are entering exciting times for retinal research with emerging genetic and imaging technologies that only a few years ago were unthinkable.

\section{Acknowledgements}

We would like to thank Dr. Matthias Gesemann for comments on the manuscript and the Swiss National Science foundation for funding. We would also like to apologize for all the publications that we could not cite due to space restrictions.

\section{Publication bibliography}

Ablain, Julien; Durand, Ellen M.; Yang, Song; Zhou, Yi; Zon, Leonard I. (2015): A CRISPR/Cas9 vector system for tissue-specific gene disruption in zebrafish. In Developmental cell 32 (6), pp. 756-764. DOI: 10.1016/j.devcel.2015.01.032.

Albadri, Shahad; Del Bene, Filippo; Revenu, Celine (2017): Genome editing using CRISPR/Cas9-based knock-in approaches in zebrafish. In Methods (San Diego, Calif.). DOI: 10.1016/j.ymeth.2017.03.005.

Allison, W. Ted; Barthel, Linda K.; Skebo, Kristina M.; Takechi, Masaki; Kawamura, Shoji; Raymond, Pamela A. (2010): Ontogeny of cone photoreceptor mosaics in zebrafish. In The Journal of comparative neurology 518 (20), pp. 4182-4195. DOI: 10.1002/cne.22447.

Allison, W. Ted; Haimberger, Theodore J.; Hawryshyn, Craig W.; Temple, Shelby E. (2004): Visual pigment composition in zebrafish: Evidence for a rhodopsin-porphyropsin interchange system. In Visual neuroscience 21 (6), pp. 945-952. DOI: $10.1017 /$ S0952523804216145.

Almeida, Alexandra D.; Boije, Henrik; Chow, Renee W.; He, Jie; Tham, Jonathan; Suzuki, Sachihiro C.; Harris, William A. (2014): Spectrum of Fates: a new approach to the study of the developing zebrafish retina. In Development (Cambridge, England) 141 (9), pp. 19711980. DOI: 10.1242/dev.104760. 
Amsterdam, A.; Burgess, S.; Golling, G.; Chen, W.; Sun, Z.; Townsend, K. et al. (1999): A large-scale insertional mutagenesis screen in zebrafish. In Genes \& development 13 (20), pp. 2713-2724.

Ando, Ryoko; Hama, Hiroshi; Yamamoto-Hino, Miki; Mizuno, Hideaki; Miyawaki, Atsushi (2002): An optical marker based on the UV-induced green-to-red photoconversion of a fluorescent protein. In Proceedings of the National Academy of Sciences of the United States of America 99 (20), pp. 12651-12656. DOI: 10.1073/pnas.202320599.

Armstrong, Gary Alan Barclay; Liao, Meijiang; You, Zhipeng; Lissouba, Alexandra; Chen, Brian Edwin; Drapeau, Pierre (2016): Homology Directed Knockin of Point Mutations in the Zebrafish tardbp and fus Genes in ALS Using the CRISPR/Cas9 System. In PloS one 11 (3), e0150188. DOI: 10.1371/journal.pone.0150188.

Asakawa, Kazuhide; Kawakami, Koichi (2008): Targeted gene expression by the Gal4-UAS system in zebrafish. In Development, growth \& differentiation 50 (6), pp. 391-399. DOI: 10.1111/j.1440-169X.2008.01044.x.

Asakawa, Kazuhide; Suster, Maximiliano L.; Mizusawa, Kanta; Nagayoshi, Saori; Kotani, Tomoya; Urasaki, Akihiro et al. (2008): Genetic dissection of neural circuits by Tol2 transposon-mediated Gal4 gene and enhancer trapping in zebrafish. In Proceedings of the National Academy of Sciences of the United States of America 105 (4), pp. 1255-1260. DOI: 10.1073/pnas.0704963105.

Auer, Thomas O.; Duroure, Karine; Cian, Anne de; Concordet, Jean-Paul; Del Bene, Filippo (2014): Highly efficient CRISPR/Cas9-mediated knock-in in zebrafish by homologyindependent DNA repair. In Genome research 24 (1), pp. 142-153. DOI: 10.1101/gr.161638.113.

Baden, Tom; Nikolaev, Anton; Esposti, Federico; Dreosti, Elena; Odermatt, Benjamin; Lagnado, Leon (2014): A synaptic mechanism for temporal filtering of visual signals. In PLoS biology 12 (10), e1001972. DOI: 10.1371/journal.pbio.1001972.

Balciunas, Darius; Davidson, Ann E.; Sivasubbu, Sridhar; Hermanson, Spencer B.; Welle, Zachary; Ekker, Stephen C. (2004): Enhancer trapping in zebrafish using the Sleeping Beauty transposon. In BMC genomics 5 (1), p. 62. DOI: 10.1186/1471-2164-5-62.

Balciuniene, Jorune; Nagelberg, Danielle; Walsh, Kathleen T.; Camerota, Diana; Georlette, Daphné; Biemar, Frédéric et al. (2013): Efficient disruption of Zebrafish genes using a Gal4containing gene trap. In BMC genomics 14, p. 619. DOI: 10.1186/1471-2164-14-619.

Biehlmaier, Oliver; Neuhauss, Stephan C. F.; Kohler, Konrad (2003): Synaptic plasticity and functionality at the cone terminal of the developing zebrafish retina. In Journal of neurobiology 56 (3), pp. 222-236. DOI: 10.1002/neu.10243. 
Bilotta, J.; Saszik, S.; Sutherland, S. E. (2001): Rod contributions to the electroretinogram of the dark-adapted developing zebrafish. In Developmental dynamics : an official publication of the American Association of Anatomists 222 (4), pp. 564-570. DOI: 10.1002/dvdy.1188.

Bitinaite, J.; Wah, D. A.; Aggarwal, A. K.; Schildkraut, I. (1998): Fokl dimerization is required for DNA cleavage. In Proceedings of the National Academy of Sciences of the United States of America 95 (18), pp. 10570-10575.

Branchek, T. (1984): The development of photoreceptors in the zebrafish, brachydanio rerio. II. Function. In The Journal of comparative neurology 224 (1), pp. 116-122. DOI: 10.1002/cne.902240110.

Branchek, T.; Bremiller, R. (1984): The development of photoreceptors in the zebrafish, Brachydanio rerio. I. Structure. In The Journal of comparative neurology 224 (1), pp. 107115. DOI: $10.1002 /$ cne.902240109.

Branda, Catherine S.; Dymecki, Susan M. (2004): Talking about a revolution: The impact of site-specific recombinases on genetic analyses in mice. In Developmental cell 6 (1), pp. 7-28. Brockerhoff, S. E.; Hurley, J. B.; Janssen-Bienhold, U.; Neuhauss, S. C.; Driever, W.; Dowling, J. E. (1995): A behavioral screen for isolating zebrafish mutants with visual system defects. In Proceedings of the National Academy of Sciences of the United States of America 92 (23), pp. 10545-10549.

Brockerhoff, S. E.; Hurley, J. B.; Niemi, G. A.; Dowling, J. E. (1997): A new form of inherited red-blindness identified in zebrafish. In The Journal of neuroscience : the official journal of the Society for Neuroscience 17 (11), pp. 4236-4242.

Brockerhoff, Susan E.; Rieke, Fred; Matthews, Hugh R.; Taylor, Michael R.; Kennedy, Breandan; Ankoudinova, Irina et al. (2003): Light stimulates a transducin-independent increase of cytoplasmic $\mathrm{Ca} 2+$ and suppression of current in cones from the zebrafish mutant nof. In The Journal of neuroscience : the official journal of the Society for Neuroscience 23 (2), pp. 470-480.

Broussard, Gerard J.; Liang, Ruqiang; Tian, Lin (2014): Monitoring activity in neural circuits with genetically encoded indicators. In Frontiers in molecular neuroscience 7, p. 97. DOI: 10.3389/fnmol.2014.00097.

Burger, Alexa; Lindsay, Helen; Felker, Anastasia; Hess, Christopher; Anders, Carolin; Chiavacci, Elena et al. (2016): Maximizing mutagenesis with solubilized CRISPR-Cas9 ribonucleoprotein complexes. In Development (Cambridge, England) 143 (11), pp. 20252037. DOI: 10.1242/dev.134809.

Burrill, J. D.; Easter, S. S., JR (1994): Development of the retinofugal projections in the embryonic and larval zebrafish (Brachydanio rerio). In The Journal of comparative neurology 346 (4), pp. 583-600. DOI: 10.1002/cne.903460410. 
Cameron, David A. (2002): Mapping absorbance spectra, cone fractions, and neuronal mechanisms to photopic spectral sensitivity in the zebrafish. In Visual neuroscience 19 (3), pp. 365-372.

Campbell, Leah J.; Willoughby, John J.; Jensen, Abbie M. (2012): Two types of Tet-On transgenic lines for doxycycline-inducible gene expression in zebrafish rod photoreceptors and a gateway-based tet-on toolkit. In PloS one 7 (12), e51270. DOI:

10.1371/journal.pone.0051270.

Clark, D. T. (1981): Visual Responses in Developing Zebrafish (Brachydanio Rerio). Ph.D. Thesis. University of Oregon, Eugene, OR.

Collery, Ross F.; Cederlund, Maria L.; Kennedy, Breandan N. (2013): Transgenic zebrafish expressing mutant human RETGC-1 exhibit aberrant cone and rod morphology. In Experimental eye research 108, pp. 120-128. DOI: 10.1016/j.exer.2013.01.003.

Connaughton, V. P. (2011): Bipolar cells in the zebrafish retina. In Visual neuroscience 28 (1), pp. 77-93. DOI: 10.1017/S0952523810000295.

Connaughton, V. P.; Graham, D.; Nelson, R. (2004): Identification and morphological classification of horizontal, bipolar, and amacrine cells within the zebrafish retina. In The Journal of comparative neurology 477 (4), pp. 371-385. DOI: 10.1002/cne.20261.

Connaughton, Victoria P.; Nelson, Ralph; Bender, Anna M. (2008): Electrophysiological evidence of GABAA and GABAC receptors on zebrafish retinal bipolar cells. In Visual neuroscience 25 (2), pp. 139-153. DOI: 10.1017/S0952523808080322.

Curado, Silvia; Anderson, Ryan M.; Jungblut, Benno; Mumm, Jeff; Schroeter, Eric; Stainier, Didier Y. R. (2007): Conditional targeted cell ablation in zebrafish: a new tool for regeneration studies. In Developmental dynamics : an official publication of the American Association of Anatomists 236 (4), pp. 1025-1035. DOI: 10.1002/dvdy.21100.

Deml, Brett; Kariminejad, Ariana; Borujerdi, Razieh H. R.; Muheisen, Sanaa; Reis, Linda M.; Semina, Elena V. (2015): Mutations in MAB21L2 result in ocular Coloboma, microcornea and cataracts. In PLoS genetics 11 (2), e1005002. DOI: 10.1371/journal.pgen.1005002.

D'Orazi, Florence D.; Zhao, Xiao-Feng; Wong, Rachel O.; Yoshimatsu, Takeshi (2016): Mismatch of Synaptic Patterns between Neurons Produced in Regeneration and during Development of the Vertebrate Retina. In Current biology : CB 26 (17), pp. 2268-2279. DOI: 10.1016/j.cub.2016.06.063.

Doyon, Yannick; McCammon, Jasmine M.; Miller, Jeffrey C.; Faraji, Farhoud; Ngo, Catherine; Katibah, George E. et al. (2008): Heritable targeted gene disruption in zebrafish using designed zinc-finger nucleases. In Nature biotechnology 26 (6), pp. 702-708. DOI: $10.1038 / \mathrm{nbt1409}$. 
Draper, Bruce W.; McCallum, Claire M.; Stout, Jennifer L.; Slade, Ann J.; Moens, Cecilia B. (2004): A high-throughput method for identifying N-ethyl-N-nitrosourea (ENU)-induced point mutations in zebrafish. In Methods in cell biology 77, pp. 91-112.

Dreosti, Elena; Odermatt, Benjamin; Dorostkar, Mario M.; Lagnado, Leon (2009): A genetically encoded reporter of synaptic activity in vivo. In Nature methods 6 (12), pp. 883889. DOI: 10.1038/nmeth.1399.

Driever, W.; Solnica-Krezel, L.; Schier, A. F.; Neuhauss, S. C.; Malicki, J.; Stemple, D. L. et al. (1996): A genetic screen for mutations affecting embryogenesis in zebrafish. In Development (Cambridge, England) 123, pp. 37-46.

Easter, S. S.; Nicola, G. N. (1996): The development of vision in the zebrafish (Danio rerio). In Developmental biology 180 (2), pp. 646-663.

Emran, Farida; Rihel, Jason; Adolph, Alan R.; Wong, Kwoon Y.; Kraves, Sebastian; Dowling, John E. (2007): OFF ganglion cells cannot drive the optokinetic reflex in zebrafish. In Proceedings of the National Academy of Sciences of the United States of America 104 (48), pp. 19126-19131. DOI: 10.1073/pnas.0709337104.

Emran, Farida; Rihel, Jason; Dowling, John E. (2008): A behavioral assay to measure responsiveness of zebrafish to changes in light intensities. In Journal of visualized experiments : JoVE (20). DOI: 10.3791/923.

Enright, Jennifer M.; Toomey, Matthew B.; Sato, Shin-ya; Temple, Shelby E.; Allen, James R.; Fujiwara, Rina et al. (2015): Cyp27c1 Red-Shifts the Spectral Sensitivity of Photoreceptors by Converting Vitamin A1 into A2. In Current biology : CB 25 (23), pp. 3048-3057. DOI: 10.1016/j.cub.2015.10.018.

Fadool, J. M.; Hartl, D. L.; Dowling, J. E. (1998): Transposition of the mariner element from Drosophila mauritiana in zebrafish. In Proceedings of the National Academy of Sciences of the United States of America 95 (9), pp. 5182-5186.

Fadool, James M. (2003): Development of a rod photoreceptor mosaic revealed in transgenic zebrafish. In Developmental biology 258 (2), pp. 277-290.

Feil, R.; Wagner, J.; Metzger, D.; Chambon, P. (1997): Regulation of Cre recombinase activity by mutated estrogen receptor ligand-binding domains. In Biochemical and biophysical research communications 237 (3), pp. 752-757. DOI: 10.1006/bbrc.1997.7124.

Fosque, Benjamin F.; Sun, Yi; Dana, Hod; Yang, Chao-Tsung; Ohyama, Tomoko; Tadross, Michael R. et al. (2015): Neural circuits. Labeling of active neural circuits in vivo with designed calcium integrators. In Science (New York, N.Y.) 347 (6223), pp. 755-760. DOI: 10.1126/science.1260922.

Gagnon, James A.; Valen, Eivind; Thyme, Summer B.; Huang, Peng; Akhmetova, Laila; Ahkmetova, Laila et al. (2014): Efficient mutagenesis by Cas9 protein-mediated 
oligonucleotide insertion and large-scale assessment of single-guide RNAs. In PloS one 9 (5), e98186. DOI: 10.1371/journal.pone.0098186.

Gaiano, N.; Allende, M.; Amsterdam, A.; Kawakami, K.; Hopkins, N. (1996a): Highly efficient germ-line transmission of proviral insertions in zebrafish. In Proceedings of the National Academy of Sciences of the United States of America 93 (15), pp. 7777-7782.

Gaiano, N.; Amsterdam, A.; Kawakami, K.; Allende, M.; Becker, T.; Hopkins, N. (1996b): Insertional mutagenesis and rapid cloning of essential genes in zebrafish. In Nature 383 (6603), pp. 829-832. DOI: 10.1038/383829a0.

Gaj, Thomas; Gersbach, Charles A.; Barbas, Carlos F. 3rd (2013): ZFN, TALEN, and CRISPR/Cas-based methods for genome engineering. In Trends in biotechnology 31 (7), pp. 397-405. DOI: 10.1016/j.tibtech.2013.04.004.

Gasiunas, Giedrius; Barrangou, Rodolphe; Horvath, Philippe; Siksnys, Virginijus (2012): Cas9crRNA ribonucleoprotein complex mediates specific DNA cleavage for adaptive immunity in bacteria. In Proceedings of the National Academy of Sciences of the United States of America 109 (39), E2579-86. DOI: 10.1073/pnas.1208507109.

Gestri, Gaia; Link, Brian A.; Neuhauss, Stephan C. F. (2012): The visual system of zebrafish and its use to model human ocular diseases. In Developmental neurobiology 72 (3), pp. 302327. DOI: 10.1002/dneu.20919.

Gilchrist, Erin J.; O'Neil, Nigel J.; Rose, Ann M.; Zetka, Monique C.; Haughn, George W. (2006): TILLING is an effective reverse genetics technique for Caenorhabditis elegans. In BMC genomics 7, p. 262. DOI: 10.1186/1471-2164-7-262.

Glasauer, Stella M. K.; Wager, Robert; Gesemann, Matthias; Neuhauss, Stephan C. F. (2016): mglur6b:EGFP Transgenic zebrafish suggest novel functions of metabotropic glutamate signaling in retina and other brain regions. In The Journal of comparative neurology 524 (12), pp. 2363-2378. DOI: 10.1002/cne.24029.

Gnuegge, L.; Schmid, S.; Neuhauss, S. C. (2001): Analysis of the activity-deprived zebrafish mutant macho reveals an essential requirement of neuronal activity for the development of a fine-grained visuotopic map. In The Journal of neuroscience : the official journal of the Society for Neuroscience 21 (10), pp. 3542-3548.

Goldman, Daniel (2014): Muller glial cell reprogramming and retina regeneration. In Nature reviews. Neuroscience 15 (7), pp. 431-442. DOI: 10.1038/nrn3723.

Gossen, M.; Bujard, H. (1992): Tight control of gene expression in mammalian cells by tetracycline-responsive promoters. In Proceedings of the National Academy of Sciences of the United States of America 89 (12), pp. 5547-5551.

Gossen, M.; Freundlieb, S.; Bender, G.; Muller, G.; Hillen, W.; Bujard, H. (1995): Transcriptional activation by tetracyclines in mammalian cells. In Science (New York, N.Y.) 268 (5218), pp. 1766-1769. 
Gross, Jeffrey M.; Perkins, Brian D.; Amsterdam, Adam; Egaña, Ana; Darland, Tristan; Matsui, Jonathan I. et al. (2005): Identification of zebrafish insertional mutants with defects in visual system development and function. In Genetics 170 (1), pp. 245-261. DOI:

10.1534/genetics.104.039727.

Groth, A. C.; Olivares, E. C.; Thyagarajan, B.; Calos, M. P. (2000): A phage integrase directs efficient site-specific integration in human cells. In Proceedings of the National Academy of Sciences of the United States of America 97 (11), pp. 5995-6000. DOI: 10.1073/pnas.090527097.

Guarente, L.; Yocum, R. R.; Gifford, P. (1982): A GAL10-CYC1 hybrid yeast promoter identifies the GAL4 regulatory region as an upstream site. In Proceedings of the National Academy of Sciences of the United States of America 79 (23), pp. 7410-7414.

Gupta, Vikas; Poss, Kenneth D. (2012): Clonally dominant cardiomyocytes direct heart morphogenesis. In Nature 484 (7395), pp. 479-484. DOI: 10.1038/nature11045.

Habuchi, Satoshi; Ando, Ryoko; Dedecker, Peter; Verheijen, Wendy; Mizuno, Hideaki; Miyawaki, Atsushi; Hofkens, Johan (2005): Reversible single-molecule photoswitching in the GFP-like fluorescent protein Dronpa. In Proceedings of the National Academy of Sciences of the United States of America 102 (27), pp. 9511-9516. DOI: 10.1073/pnas.0500489102.

Haffter, P.; Granato, M.; Brand, M.; Mullins, M. C.; Hammerschmidt, M.; Kane, D. A. et al. (1996): The identification of genes with unique and essential functions in the development of the zebrafish, Danio rerio. In Development (Cambridge, England) 123, pp. 1-36.

Halloran, M. C.; Sato-Maeda, M.; Warren, J. T.; Su, F.; Lele, Z.; Krone, P. H. et al. (2000): Laser-induced gene expression in specific cells of transgenic zebrafish. In Development (Cambridge, England) 127 (9), pp. 1953-1960.

Hans, Stefan; Freudenreich, Dorian; Geffarth, Michaela; Kaslin, Jan; Machate, Anja; Brand, Michael (2011): Generation of a non-leaky heat shock-inducible Cre line for conditional Cre/lox strategies in zebrafish. In Developmental dynamics : an official publication of the American Association of Anatomists 240 (1), pp. 108-115. DOI: 10.1002/dvdy.22497.

Hans, Stefan; Kaslin, Jan; Freudenreich, Dorian; Brand, Michael (2009): Temporallycontrolled site-specific recombination in zebrafish. In PloS one 4 (2), e4640. DOI: 10.1371/journal.pone.0004640.

He, Jie; Zhang, Gen; Almeida, Alexandra D.; Cayouette, Michel; Simons, Benjamin D.; Harris, William A. (2012): How variable clones build an invariant retina. In Neuron 75 (5), pp. 786798. DOI: 10.1016/j.neuron.2012.06.033.

He, Mu-Dan; Zhang, Feng-Hua; Wang, Hua-Lin; Wang, Hou-Peng; Zhu, Zuo-Yan; Sun, YongHua (2015): Efficient ligase 3-dependent microhomology-mediated end joining repair of DNA double-strand breaks in zebrafish embryos. In Mutation research 780, pp. 86-96. DOI: 10.1016/j.mrfmmm.2015.08.004. 
Hirsh, D.; Vanderslice, R. (1976): Temperature-sensitive developmental mutants of Caenorhabditis elegans. In Developmental biology 49 (1), pp. 220-235.

Howe, Kerstin; Clark, Matthew D.; Torroja, Carlos F.; Torrance, James; Berthelot, Camille; Muffato, Matthieu et al. (2013): The zebrafish reference genome sequence and its relationship to the human genome. In Nature 496 (7446), pp. 498-503. DOI: $10.1038 /$ nature12111.

Hruscha, Alexander; Krawitz, Peter; Rechenberg, Alexandra; Heinrich, Verena; Hecht, Jochen; Haass, Christian; Schmid, Bettina (2013): Efficient CRISPR/Cas9 genome editing with low off-target effects in zebrafish. In Development (Cambridge, England) 140 (24), pp. 4982 4987. DOI: 10.1242/dev.099085.

http://tol2kit.genetics.utah.edu/index.php/Main_Page: Tol2kit. Available online at http://tol2kit.genetics.utah.edu/index.php/Main_Page, checked on 3/31/2017.

https://www.sanger.ac.uk/sanger/Zebrafish_Zmpbrowse: Wellcome Trust Sanger Institute. Available online at https://www.sanger.ac.uk/sanger/Zebrafish_Zmpbrowse, checked on 2/13/2017.

Hu, Gui; Goll, Mary G.; Fisher, Shannon (2011): PhiC31 integrase mediates efficient cassette exchange in the zebrafish germline. In Developmental dynamics : an official publication of the American Association of Anatomists 240 (9), pp. 2101-2107. DOI: 10.1002/dvdy.22699.

Hu, M.; Easter, S. S. (1999): Retinal neurogenesis: the formation of the initial central patch of postmitotic cells. In Developmental biology 207 (2), pp. 309-321. DOI: 10.1006/dbio.1998.9031.

Huang, Chiu-Ju; Jou, Tzuu-Shuh; Ho, Yi-Lwun; Lee, Wu-Hsun; Jeng, Yu-Ting; Hsieh, Fong-Jou; Tsai, Huai-Jen (2005): Conditional expression of a myocardium-specific transgene in zebrafish transgenic lines. In Developmental dynamics : an official publication of the American Association of Anatomists 233 (4), pp. 1294-1303. DOI: 10.1002/dvdy.20485.

Huang, Peng; Zhu, Zuoyan; Lin, Shuo; Zhang, Bo (2012): Reverse genetic approaches in zebrafish. In Journal of genetics and genomics = Yi chuan xue bao 39 (9), pp. 421-433. DOI: 10.1016/j.jgg.2012.07.004.

Huber-Reggi, Sabina P.; Mueller, Kaspar P.; Neuhauss, Stephan C F (2013): Analysis of optokinetic response in zebrafish by computer-based eye tracking. In Methods in molecular biology (Clifton, N.J.) 935, pp. 139-160. DOI: 10.1007/978-1-62703-080-9_10.

Hughes, A.; Saszik, S.; Bilotta, J.; Demarco, P. J.; Patterson, W. F. (1998): Cone contributions to the photopic spectral sensitivity of the zebrafish ERG. In Visual neuroscience 15 (6), pp. 1029-1037.

Hwang, Woong Y.; Fu, Yanfang; Reyon, Deepak; Maeder, Morgan L.; Kaini, Prakriti; Sander, Jeffry D. et al. (2013): Heritable and precise zebrafish genome editing using a CRISPR-Cas system. In PloS one 8 (7), e68708. DOI: 10.1371/journal.pone.0068708. 
Jao, Li-En; Wente, Susan R.; Chen, Wenbiao (2013): Efficient multiplex biallelic zebrafish genome editing using a CRISPR nuclease system. In Proceedings of the National Academy of Sciences of the United States of America 110 (34), pp. 13904-13909. DOI: 10.1073/pnas.1308335110.

Jinek, Martin; Chylinski, Krzysztof; Fonfara, Ines; Hauer, Michael; Doudna, Jennifer A.; Charpentier, Emmanuelle (2012): A programmable dual-RNA-guided DNA endonuclease in adaptive bacterial immunity. In Science (New York, N.Y.) 337 (6096), pp. 816-821. DOI: 10.1126/science.1225829.

Kawakami, K.; Shima, A.; Kawakami, N. (2000): Identification of a functional transposase of the Tol 2 element, an Ac-like element from the Japanese medaka fish, and its transposition in the zebrafish germ lineage. In Proceedings of the National Academy of Sciences of the United States of America 97 (21), pp. 11403-11408. DOI: 10.1073/pnas.97.21.11403. Kawakami, Koichi (2007): Tol2: a versatile gene transfer vector in vertebrates. In Genome biology 8 Suppl 1, S7. DOI: 10.1186/gb-2007-8-s1-s7.

Kawakami, Koichi; Takeda, Hisashi; Kawakami, Noriko; Kobayashi, Makoto; Matsuda, Naoto; Mishina, Masayoshi (2004): A transposon-mediated gene trap approach identifies developmentally regulated genes in zebrafish. In Developmental cell 7 (1), pp. 133-144. DOI: 10.1016/j.devcel.2004.06.005.

Kettleborough, Ross N. W.; Busch-Nentwich, Elisabeth M.; Harvey, Steven A.; Dooley, Christopher M.; Bruijn, Ewart de; van Eeden, Freek et al. (2013): A systematic genome-wide analysis of zebrafish protein-coding gene function. In Nature 496 (7446), pp. 494-497. DOI: 10.1038/nature11992.

Kimmel, C. B.; Patterson, J.; Kimmel, R. O. (1974): The development and behavioral characteristics of the startle response in the zebra fish. In Developmental psychobiology 7 (1), pp. 47-60. DOI: 10.1002/dev.420070109.

Klaassen, Lauw J.; Graaff, Wim de; Van Asselt, Jorrit B; Klooster, Jan; Kamermans, Maarten (2016): Specific connectivity between photoreceptors and horizontal cells in the zebrafish retina. In Journal of neurophysiology, jn.00449.2016. DOI: 10.1152/jn.00449.2016.

Kljavin, I. J. (1987): Early development of photoreceptors in the ventral retina of the zebrafish embryo. In The Journal of comparative neurology 260 (3), pp. 461-471. DOI: 10.1002/cne.902600311.

Knopf, Franziska; Schnabel, Kristin; Haase, Christa; Pfeifer, Katja; Anastassiadis, Konstantinos; Weidinger, Gilbert (2010): Dually inducible TetON systems for tissue-specific conditional gene expression in zebrafish. In Proceedings of the National Academy of Sciences of the United States of America 107 (46), pp. 19933-19938. DOI: 10.1073/pnas.1007799107. Koga, Akihiko; Cheah, Felicia S H; Hamaguchi, Satoshi; Yeo, Gare Hoon; Chong, Samuel S. (2008): Germline transgenesis of zebrafish using the medaka Tol1 transposon system. In 
Developmental dynamics : an official publication of the American Association of Anatomists 237 (9), pp. 2466-2474. DOI: 10.1002/dvdy.21688.

Kok, Fatma O.; Shin, Masahiro; Ni, Chih-Wen; Gupta, Ankit; Grosse, Ann S.; van Impel, Andreas et al. (2015): Reverse genetic screening reveals poor correlation between morpholino-induced and mutant phenotypes in zebrafish. In Developmental cell 32 (1), pp. 97-108. DOI: 10.1016/j.devcel.2014.11.018.

Kotani, Tomoya; Nagayoshi, Saori; Urasaki, Akihiro; Kawakami, Koichi (2006): Transposonmediated gene trapping in zebrafish. In Methods (San Diego, Calif.) 39 (3), pp. 199-206. DOI: 10.1016/j.ymeth.2005.12.006.

Kwan, Kristen M.; Fujimoto, Esther; Grabher, Clemens; Mangum, Benjamin D.; Hardy, Melissa E.; Campbell, Douglas S. et al. (2007): The Tol2kit: a multisite gateway-based construction kit for Tol2 transposon transgenesis constructs. In Developmental dynamics : an official publication of the American Association of Anatomists 236 (11), pp. 3088-3099. DOI: $10.1002 / d v d y .21343$.

Le, Xiuning; Langenau, David M.; Keefe, Matthew D.; Kutok, Jeffery L.; Neuberg, Donna S.; Zon, Leonard I. (2007): Heat shock-inducible Cre/Lox approaches to induce diverse types of tumors and hyperplasia in transgenic zebrafish. In Proceedings of the National Academy of Sciences of the United States of America 104 (22), pp. 9410-9415. DOI: 10.1073/pnas.0611302104.

Li, L.; Dowling, J. E. (1997): A dominant form of inherited retinal degeneration caused by a non-photoreceptor cell-specific mutation. In Proceedings of the National Academy of Sciences of the United States of America 94 (21), pp. 11645-11650.

Li, L.; Dowling, J. E. (2000): Effects of dopamine depletion on visual sensitivity of zebrafish. In The Journal of neuroscience : the official journal of the Society for Neuroscience 20 (5), pp. 1893-1903.

Li, Yong N.; Matsui, Jonathan I.; Dowling, John E. (2009): Specificity of the horizontal cellphotoreceptor connections in the zebrafish (Danio rerio) retina. In The Journal of comparative neurology 516 (5), pp. 442-453. DOI: 10.1002/cne.22135.

Li, Yong N.; Tsujimura, Taro; Kawamura, Shoji; Dowling, John E. (2012): Bipolar cellphotoreceptor connectivity in the zebrafish (Danio rerio) retina. In The Journal of comparative neurology 520 (16), pp. 3786-3802. DOI: 10.1002/cne.23168.

Ma, E. Y.; Lewis, A.; Barabas, P.; Stearns, G.; Suzuki, S.; Krizaj, D.; Brockerhoff, S. E. (2013): Loss of $\mathrm{Pde} 6$ reduces cell body $\mathrm{Ca}(2+)$ transients within photoreceptors. In Cell death \& disease 4, e797. DOI: 10.1038/cddis.2013.332.

MacDonald, Ryan B.; Kashikar, Nachiket D.; Lagnado, Leon; Harris, William A. (2016): A Novel Tool to Measure Extracellular Glutamate in the Zebrafish Nervous System In Vivo. In Zebrafish. DOI: 10.1089/zeb.2016.1385. 
Makhankov, Yuri V.; Rinner, Oliver; Neuhauss, Stephan C F (2004): An inexpensive device for non-invasive electroretinography in small aquatic vertebrates. In Journal of neuroscience methods 135 (1-2), pp. 205-210. DOI: 10.1016/j.jneumeth.2003.12.015.

Malicki, J.; Neuhauss, S. C.; Schier, A. F.; Solnica-Krezel, L.; Stemple, D. L.; Stainier, D. Y. et al. (1996): Mutations affecting development of the zebrafish retina. In Development (Cambridge, England) 123, pp. 263-273.

Malicki, J.; Pooranachandran, N.; Nikolaev, A.; Fang, X.; Avanesov, A. (2016): Analysis of the retina in the zebrafish model. In Methods in cell biology 134, pp. 257-334. DOI:

10.1016/bs.mcb.2016.04.017.

Malicki, Jarema; Jo, Hakryul; Wei, Xiangyun; Hsiung, Monica; Pujic, Zac (2002): Analysis of gene function in the zebrafish retina. In Methods (San Diego, Calif.) 28 (4), pp. 427-438.

Marvin, Jonathan S.; Borghuis, Bart G.; Tian, Lin; Cichon, Joseph; Harnett, Mark T.;

Akerboom, Jasper et al. (2013): An optimized fluorescent probe for visualizing glutamate neurotransmission. In Nature methods 10 (2), pp. 162-170. DOI: 10.1038/nmeth.2333.

Masland, Richard H. (2012): The tasks of amacrine cells. In Visual neuroscience 29 (1), pp. 39.

McCallum, C. M.; Comai, L.; Greene, E. A.; Henikoff, S. (2000): Targeted screening for induced mutations. In Nature biotechnology 18 (4), pp. 455-457. DOI: 10.1038/74542.

Meng, Xiangdong; Noyes, Marcus B.; Zhu, Lihua J.; Lawson, Nathan D.; Wolfe, Scot A. (2008): Targeted gene inactivation in zebrafish using engineered zinc-finger nucleases. In Nature biotechnology 26 (6), pp. 695-701. DOI: 10.1038/nbt1398.

Miesfeld, Joel B.; Gestri, Gaia; Clark, Brian S.; Flinn, Michael A.; Poole, Richard J.; Bader, Jason R. et al. (2015): Yap and Taz regulate retinal pigment epithelial cell fate. In Development (Cambridge, England) 142 (17), pp. 3021-3032. DOI: 10.1242/dev.119008.

Miyawaki, A.; Griesbeck, O.; Heim, R.; Tsien, R. Y. (1999): Dynamic and quantitative Ca2+ measurements using improved cameleons. In Proceedings of the National Academy of Sciences of the United States of America 96 (5), pp. 2135-2140.

Montague, Tessa G.; Cruz, José M.; Gagnon, James A.; Church, George M.; Valen, Eivind (2014): CHOPCHOP: a CRISPR/Cas9 and TALEN web tool for genome editing. In Nucleic acids research 42 (Web Server issue), W401-7. DOI: 10.1093/nar/gku410.

Montgomery, Jacob E.; Parsons, Michael J.; Hyde, David R. (2010): A novel model of retinal ablation demonstrates that the extent of rod cell death regulates the origin of the regenerated zebrafish rod photoreceptors. In The Journal of comparative neurology 518 (6), pp. 800-814. DOI: 10.1002/cne.22243.

Moore, Finola E.; Reyon, Deepak; Sander, Jeffry D.; Martinez, Sarah A.; Blackburn, Jessica S.; Khayter, Cyd et al. (2012): Improved somatic mutagenesis in zebrafish using transcription 
activator-like effector nucleases (TALENs). In PloS one 7 (5), e37877. DOI:

10.1371/journal.pone.0037877.

Moreno-Mateos, Miguel A.; Vejnar, Charles E.; Beaudoin, Jean-Denis; Fernandez, Juan P.; Mis, Emily K.; Khokha, Mustafa K.; Giraldez, Antonio J. (2015): CRISPRscan: designing highly efficient sgRNAs for CRISPR-Cas9 targeting in vivo. In Nature methods 12 (10), pp. 982-988. DOI: $10.1038 /$ nmeth.3543.

Mosimann, Christian; Puller, Ann-Christin; Lawson, Katy L.; Tschopp, Patrick; Amsterdam, Adam; Zon, Leonard I. (2013): Site-directed zebrafish transgenesis into single landing sites with the phic31 integrase system. In Developmental dynamics : an official publication of the American Association of Anatomists 242 (8), pp. 949-963. DOI: 10.1002/dvdy.23989.

Mullins, M. C.; Hammerschmidt, M.; Haffter, P.; Nüsslein-Volhard, C. (1994): Large-scale mutagenesis in the zebrafish: in search of genes controlling development in a vertebrate. In Current biology : CB 4 (3), pp. 189-202.

Muto, Akira; Orger, Michael B.; Wehman, Ann M.; Smear, Matthew C.; Kay, Jeremy N.; PageMcCaw, Patrick S. et al. (2005): Forward genetic analysis of visual behavior in zebrafish. In PLoS genetics 1 (5), e66. DOI: 10.1371/journal.pgen.0010066.

Nagayoshi, Saori; Hayashi, Eriko; Abe, Gembu; Osato, Naoki; Asakawa, Kazuhide; Urasaki, Akihiro et al. (2008): Insertional mutagenesis by the Tol2 transposon-mediated enhancer trap approach generated mutations in two developmental genes: tcf7 and synembryn-like. In Development (Cambridge, England) 135 (1), pp. 159-169. DOI: 10.1242/dev.009050.

Nakai, J.; Ohkura, M.; Imoto, K. (2001): A high signal-to-noise $\mathrm{Ca}(2+)$ probe composed of a single green fluorescent protein. In Nature biotechnology 19 (2), pp. 137-141. DOI: $10.1038 / 84397$.

Nasevicius, A.; Ekker, S. C. (2000): Effective targeted gene 'knockdown' in zebrafish. In Nature genetics 26 (2), pp. 216-220. DOI: 10.1038/79951.

Nawrocki, L.; Bremiller, R.; Streisinger, G.; Kaplan, M. (1985): Larval and adult visual pigments of the zebrafish, Brachydanio rerio. In Vision research 25 (11), pp. 1569-1576.

Neuhauss, S. C.; Biehlmaier, O.; Seeliger, M. W.; Das, T.; Kohler, K.; Harris, W. A.; Baier, H. (1999): Genetic disorders of vision revealed by a behavioral screen of 400 essential loci in zebrafish. In The Journal of neuroscience : the official journal of the Society for Neuroscience 19 (19), pp. 8603-8615.

Nusslein-Volhard, C.; Wieschaus, E. (1980): Mutations affecting segment number and polarity in Drosophila. In Nature 287 (5785), pp. 795-801.

Oleykowski, C. A.; Bronson Mullins, C R; Godwin, A. K.; Yeung, A. T. (1998): Mutation detection using a novel plant endonuclease. In Nucleic acids research 26 (20), pp. 45974602. 
Pan, Y. Albert; Freundlich, Tom; Weissman, Tamily A.; Schoppik, David; Wang, X. Cindy; Zimmerman, Steve et al. (2013): Zebrabow: multispectral cell labeling for cell tracing and lineage analysis in zebrafish. In Development (Cambridge, England) 140 (13), pp. 2835-2846. DOI: 10.1242/dev.094631.

Pan, Y. Albert; Livet, Jean; Sanes, Joshua R.; Lichtman, Jeff W.; Schier, Alexander F. (2011): Multicolor Brainbow imaging in zebrafish. In Cold Spring Harbor protocols 2011 (1), pdb.prot5546. DOI: 10.1101/pdb.prot5546.

Patton, E. E.; Zon, L. I. (2001): The art and design of genetic screens: zebrafish. In Nature reviews. Genetics 2 (12), pp. 956-966. DOI: 10.1038/35103567.

Pavletich, N. P.; Pabo, C. O. (1991): Zinc finger-DNA recognition: crystal structure of a Zif268DNA complex at 2.1 A. In Science (New York, N.Y.) 252 (5007), pp. 809-817.

Peterson, R. E.; Fadool, J. M.; McClintock, J.; Linser, P. J. (2001): Müller cell differentiation in the zebrafish neural retina: evidence of distinct early and late stages in cell maturation. In The Journal of comparative neurology 429 (4), pp. 530-540.

Quach, Helen Ngoc Bao; Tao, Shijie; Vrljicak, Pavle; Joshi, Adita; Ruan, Hua; Sukumaran, Rashmi et al. (2015): A Multifunctional Mutagenesis System for Analysis of Gene Function in Zebrafish. In G3 (Bethesda, Md.) 5 (6), pp. 1283-1299. DOI: 10.1534/g3.114.015842.

Raymond, P. A.; Barthel, L. K.; Curran, G. A. (1995): Developmental patterning of rod and cone photoreceptors in embryonic zebrafish. In The Journal of comparative neurology 359 (4), pp. 537-550. DOI: 10.1002/cne.903590403.

Raz, E.; van Luenen, H G; Schaerringer, B.; Plasterk, R. H.; Driever, W. (1998): Transposition of the nematode Caenorhabditis elegans Tc3 element in the zebrafish Danio rerio. In Current biology : $C B 8$ (2), pp. 82-88.

Rinner, Oliver; Rick, Jens M.; Neuhauss, Stephan C F (2005): Contrast sensitivity, spatial and temporal tuning of the larval zebrafish optokinetic response. In Investigative ophthalmology \& visual science 46 (1), pp. 137-142. DOI: 10.1167/iovs.04-0682.

Roberts, Jennifer Anne; Miguel-Escalada, Irene; Slovik, Katherine Joan; Walsh, Kathleen Theodora; Hadzhiev, Yavor; Sanges, Remo et al. (2014): Targeted transgene integration overcomes variability of position effects in zebrafish. In Development (Cambridge, England) 141 (3), pp. 715-724. DOI: 10.1242/dev.100347.

Robinson, J.; Schmitt, E. A.; Hárosi, F. I.; Reece, R. J.; Dowling, J. E. (1993): Zebrafish ultraviolet visual pigment: absorption spectrum, sequence, and localization. In Proceedings of the National Academy of Sciences of the United States of America 90 (13), pp. 6009-6012.

Robles, Estuardo; Laurell, Eva; Baier, Herwig (2014): The retinal projectome reveals brainarea-specific visual representations generated by ganglion cell diversity. In Current biology : CB 24 (18), pp. 2085-2096. DOI: 10.1016/j.cub.2014.07.080. 
Rosa, Juliana M.; Ruehle, Sabine; Ding, Huayu; Lagnado, Leon (2016): Crossover Inhibition Generates Sustained Visual Responses in the Inner Retina. In Neuron 90 (2), pp. 308-319. DOI: 10.1016/j.neuron.2016.03.015.

Sadowski, I.; Ma, J.; Triezenberg, S.; Ptashne, M. (1988): GAL4-VP16 is an unusually potent transcriptional activator. In Nature 335 (6190), pp. 563-564. DOI: 10.1038/335563a0.

Saszik, S.; Bilotta, J.; Givin, C. M. (1999): ERG assessment of zebrafish retinal development. In Visual neuroscience 16 (5), pp. 881-888.

Sauer, B. (1987): Functional expression of the cre-lox site-specific recombination system in the yeast Saccharomyces cerevisiae. In Molecular and cellular biology 7 (6), pp. 2087-2096.

Schmitt, E. A.; Dowling, J. E. (1994): Early eye morphogenesis in the zebrafish, Brachydanio rerio. In The Journal of comparative neurology 344 (4), pp. 532-542. DOI:

10.1002/cne.903440404.

Seeliger, Mathias W.; Rilk, Albrecht; Neuhauss, Stephan C. F. (2002): Ganzfeld ERG in zebrafish larvae. In Documenta ophthalmologica. Advances in ophthalmology 104 (1), pp. 57-68.

Solnica-Krezel, L.; Schier, A. F.; Driever, W. (1994): Efficient recovery of ENU-induced mutations from the zebrafish germline. In Genetics 136 (4), pp. 1401-1420.

Suster, Maximiliano L.; Abe, Gembu; Schouw, Anders; Kawakami, Koichi (2011): Transposonmediated BAC transgenesis in zebrafish. In Nature protocols 6 (12), pp. 1998-2021. DOI: 10.1038/nprot.2011.416.

Suzuki, Sachihiro C.; Bleckert, Adam; Williams, Philip R.; Takechi, Masaki; Kawamura, Shoji; Wong, Rachel O. L. (2013): Cone photoreceptor types in zebrafish are generated by symmetric terminal divisions of dedicated precursors. In Proceedings of the National Academy of Sciences of the United States of America 110 (37), pp. 15109-15114. DOI: 10.1073/pnas.1303551110.

Tang, Xia; Gao, Jianan; Jia, Xinling; Zhao, Wencao; Zhang, Yijie; Pan, Weijun; He, Jie (2017): Bipotent progenitors as embryonic origin of retinal stem cells. In The Journal of cell biology. DOI: $10.1083 / j c b .201611057$.

Thummel, Ryan; Burket, Christopher T.; Brewer, Jeffrey L.; Sarras, Michael P., JR; Li, Li; Perry, Martin et al. (2005): Cre-mediated site-specific recombination in zebrafish embryos. In Developmental dynamics : an official publication of the American Association of Anatomists 233 (4), pp. 1366-1377. DOI: 10.1002/dvdy.20475.

Thyme, Summer B.; Schier, Alexander F. (2016): Polq-Mediated End Joining Is Essential for Surviving DNA Double-Strand Breaks during Early Zebrafish Development. In Cell reports 15 (7), pp. 1611-1613. DOI: 10.1016/j.celrep.2016.04.089. 
Till, Bradley J.; Cooper, Jennifer; Tai, Thomas H.; Colowit, Peter; Greene, Elizabeth A.; Henikoff, Steven; Comai, Luca (2007): Discovery of chemically induced mutations in rice by TILLING. In BMC plant biology 7, p. 19. DOI: 10.1186/1471-2229-7-19.

Turner, D. L.; Cepko, C. L. (1987): A common progenitor for neurons and glia persists in rat retina late in development. In Nature 328 (6126), pp. 131-136. DOI: 10.1038/328131a0.

Vroman, Rozan; Klaassen, Lauw J.; Howlett, Marcus H C; Cenedese, Valentina; Klooster, Jan; Sjoerdsma, Trijntje; Kamermans, Maarten (2014): Extracellular ATP hydrolysis inhibits synaptic transmission by increasing ph buffering in the synaptic cleft. In PLoS biology 12 (5), e1001864. DOI: 10.1371/journal.pbio.1001864.

Wachter, Rebekka M.; Watkins, Jennifer L.; Kim, Hanseong (2010): Mechanistic diversity of red fluorescence acquisition by GFP-like proteins. In Biochemistry 49 (35), pp. 7417-7427. DOI: 10.1021/bi100901h.

Wan, Yinan; Almeida, Alexandra D.; Rulands, Steffen; Chalour, Naima; Muresan, Leila; Wu, Yunmin et al. (2016): The ciliary marginal zone of the zebrafish retina: clonal and time-lapse analysis of a continuously growing tissue. In Development (Cambridge, England) 143 (7), pp. 1099-1107. DOI: 10.1242/dev.133314.

Wang, Tzu-Ming; Holzhausen, Lars C.; Kramer, Richard H. (2014): Imaging an optogenetic pH sensor reveals that protons mediate lateral inhibition in the retina. In Nature neuroscience 17 (2), pp. 262-268. DOI: 10.1038/nn.3627.

West, Megan C.; Campbell, Leah J.; Willoughby, John J.; Jensen, Abbie M. (2014): Two types of transgenic lines for doxycycline-inducible, cell-specific gene expression in zebrafish ultraviolet cone photoreceptors. In Gene expression patterns : GEP 14 (2), pp. 96-104. DOI: 10.1016/j.gep.2014.01.002.

Wienholds, Erno; Schulte-Merker, Stefan; Walderich, Brigitte; Plasterk, Ronald H A (2002): Target-selected inactivation of the zebrafish rag1 gene. In Science (New York, N.Y.) 297 (5578), pp. 99-102. DOI: 10.1126/science.1071762.

Wienholds, Erno; van Eeden, Freek; Kosters, Marit; Mudde, Josine; Plasterk, Ronald H A; Cuppen, Edwin (2003): Efficient target-selected mutagenesis in zebrafish. In Genome research 13 (12), pp. 2700-2707. DOI: 10.1101/gr.1725103.

Winkler, Sylke; Schwabedissen, Anja; Backasch, Dana; Bökel, Christian; Seidel, Claudia; Bönisch, Stefanie et al. (2005): Target-selected mutant screen by TILLING in Drosophila. In Genome research 15 (5), pp. 718-723. DOI: 10.1101/gr.3721805.

Yin, Linlin; Maddison, Lisette A.; Li, Mingyu; Kara, Nergis; LaFave, Matthew C.; Varshney, Gaurav K. et al. (2015): Multiplex Conditional Mutagenesis Using Transgenic Expression of Cas9 and sgRNAs. In Genetics 200 (2), pp. 431-441. DOI: 10.1534/genetics.115.176917. 
Zang, Jingjing; Keim, Jennifer; Kastenhuber, Edda; Gesemann, Matthias; Neuhauss, Stephan C. F. (2015): Recoverin depletion accelerates cone photoresponse recovery. In Open biology 5 (8). DOI: 10.1098/rsob.150086.

Zhang, Rong-wei; Wei, Hong-ping; Xia, Yi-meng; Du, Jiu-lin (2010): Development of light response and GABAergic excitation-to-inhibition switch in zebrafish retinal ganglion cells. In The Journal of physiology 588 (Pt 14), pp. 2557-2569. DOI: 10.1113/jphysiol.2010.187088.

Zhao, Xiao-Feng; Ellingsen, Staale; Fjose, Anders (2009): Labelling and targeted ablation of specific bipolar cell types in the zebrafish retina. In BMC neuroscience 10, p. 107. DOI:

10.1186/1471-2202-10-107.

\section{Figures}

Figure 1 
a


Retinal Pigment Epithelium

Outer Nuclear Layer

Outer Plexiform Layer

Inner Nuclear Layer

Inner Plexiform Layer

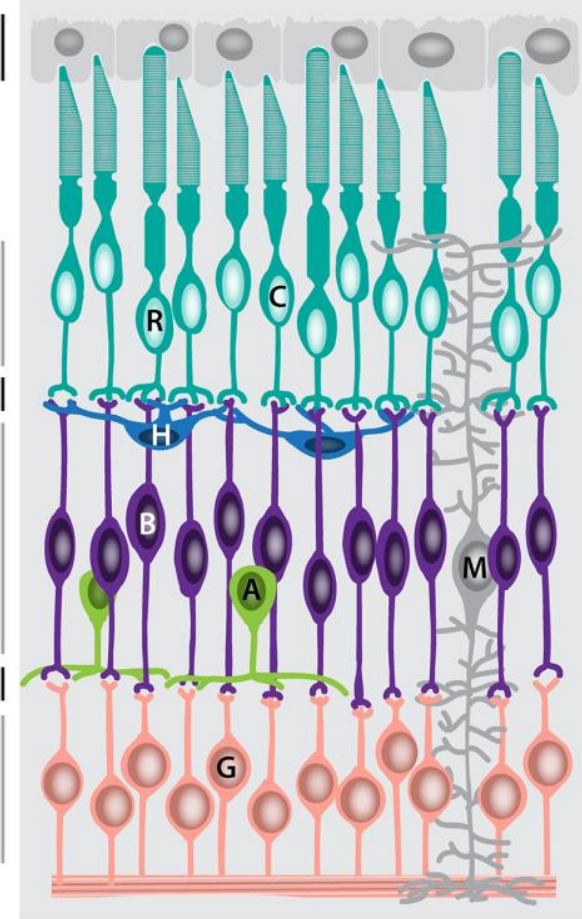

\section{Zebrafish retinal anatomy.}

Zebrafish possess a vertebrate type retina. a) Already at 5 days post fertilization, the larval retina is considered fully functional and consists of three nuclear and two synaptic layers, typical for a canonical vertebrate retina. b) Section of an adult zebrafish retina (left) with a schematic illustration (right) depicting retinal organization. The outer nuclear layer contains nuclei of rod and cone photoreceptors. Photoreceptors transmit the signal to interneurons called bipolar cells whose nuclei are located in the inner nuclear layer, together with cell bodies or horizontal and amacrine cells. Bipolar cells in turn project to ganglion cells, the retinal output cells. Horizontal and amacrine cells are inhibitory interneurons laterally modulating the signal in the synaptic layers, outer and inner plexiform layer respectively. Müller cells are the main glia cell type in the zebrafish retina. They span from the optic nerve layer to the basal end of photoreceptor inner segments. R Rods, $\mathrm{C} \mathrm{Cones,} \mathrm{H}$ Horizontal cell, B Bipolar cell, A Amacrine cell, G Ganglion cell, M Müller glia cell. 
Figure 2:

a Tol2 transgenesis

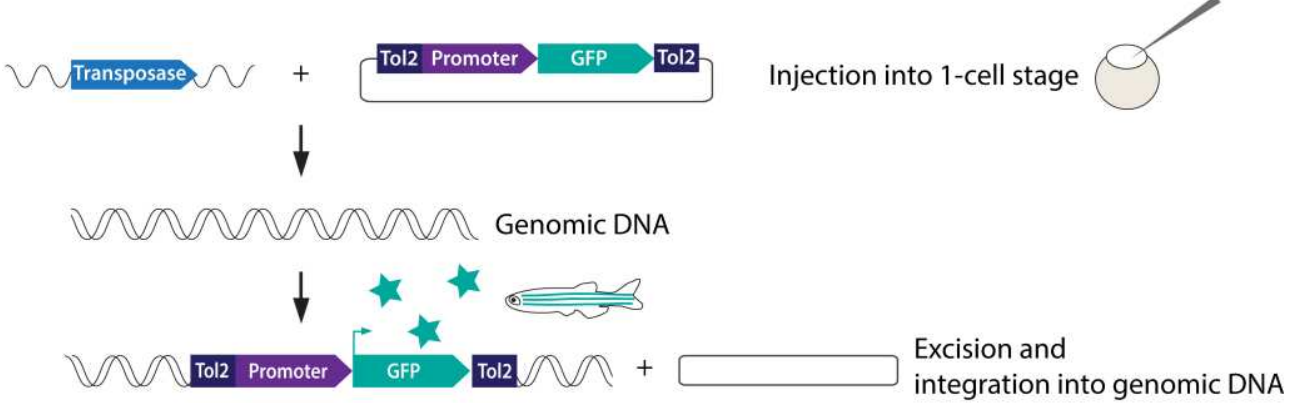

b phiC31-mediated site specific transgenesis

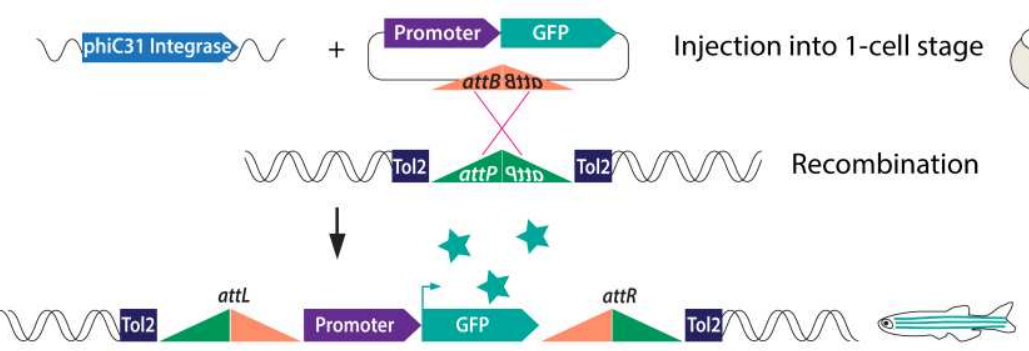

c

Gal4/UAS

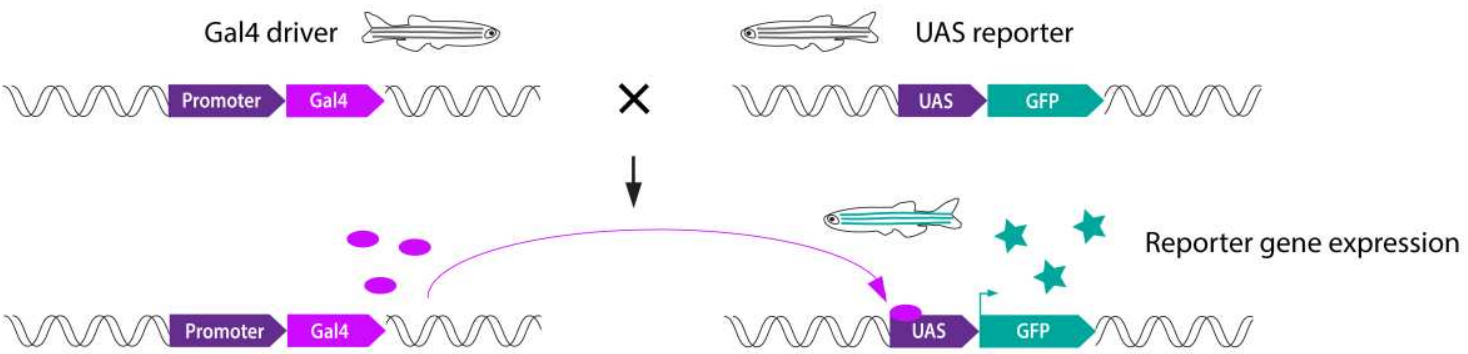

d Cre/Lox

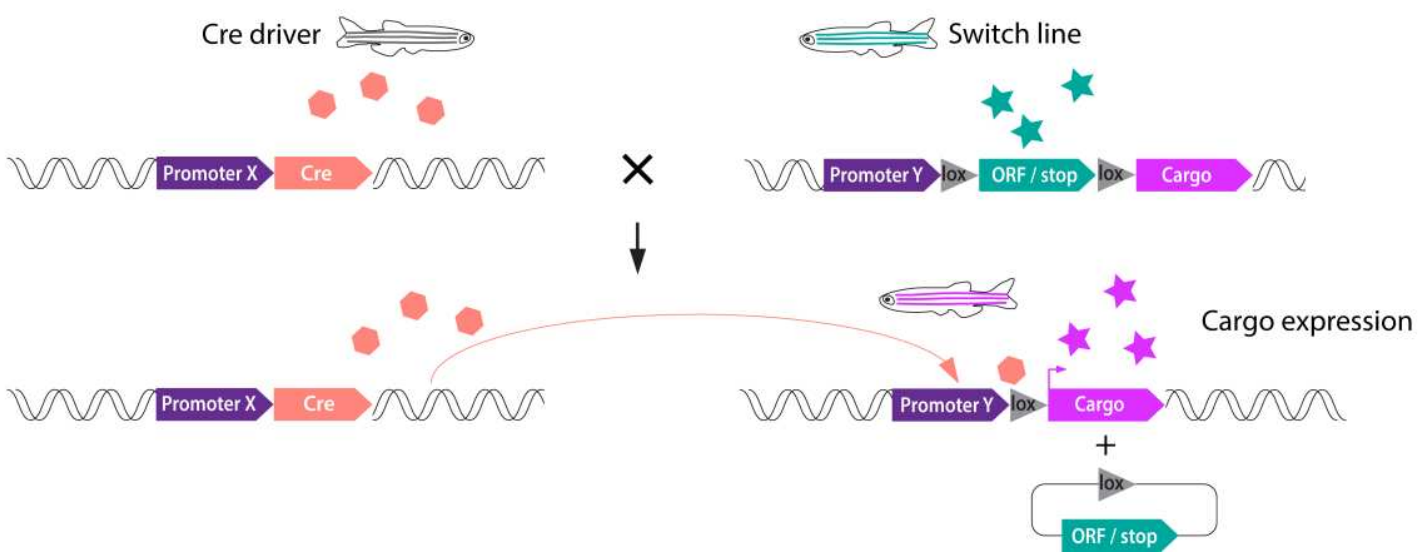

Schematic illustration of transgenesis approaches used in zebrafish. 
a) Tol2-mediated transgenesis has become the standard technique to insert exogenous DNA into the zebrafish genome. Transposase mRNA is injected together with a donor plasmid containing a Tol2 site flanked tissue specific promotor upstream of a reporter gene. Transposase mediated excision of the cassette from the plasmid and integration into the genomic DNA occurs in a randomized way. b) Random or multiple integrations into the genome can be bypassed by using the phiC31 system. Zebrafish lines carrying a single landing (attP) site can be injected with phiC31 encoding mRNA and a donor plasmid containing a reporter gene (here GFP) downstream of a promoter and an $a t t B$ site. A single integration event occurs at the attP site, catalyzed by the phiC31 integrase. The introduction of the cassette results in GFP expression in all cells with promoter activity. c) The Gal4/ Upstream activation system (UAS) is a dual transgenesis system where a Gal4 driver line is crossed to a UAS reporter line. The Gal4 driver fish expresses Gal4 under a tissue specific promoter. The UAS reporter harbors a transgene in which a reporter gene (here GFP) is downstream of the UAS. If driver and reporter fish are crossed, all cells of the progeny with promoter activity express the reporter gene, due to specific binding of Gal4 transcription activating protein to the UAS enhancer. d) Cre/Lox constitutes another powerful dual transgenesis system, where a Cre driver line is crossed to a switch line. The cre driver transgenically expresses Cre recombinase under the control of promoter $X$ (e.g. a ubiquitous promotor like ubiquitin). The switch line contains a transgene of a promoter $Y$ upstream of a floxed ORF (e.g. GFP or a stop cassette) and a cargo (e.g. RFP). By crossing driver and switch lines, progeny will show cargo (RFP) expression in cells with promoter $Y$ activity, due to Cre mediated recombination of the floxed ORF.

Figure 3:

a

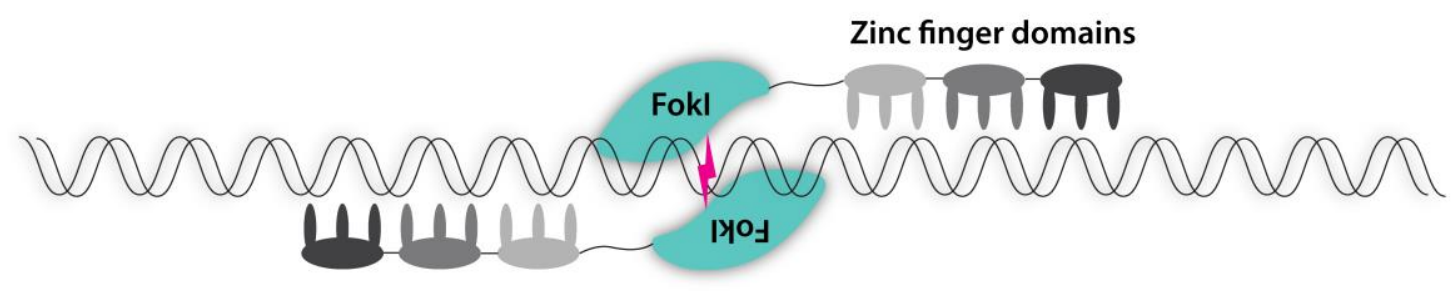

b TALENS

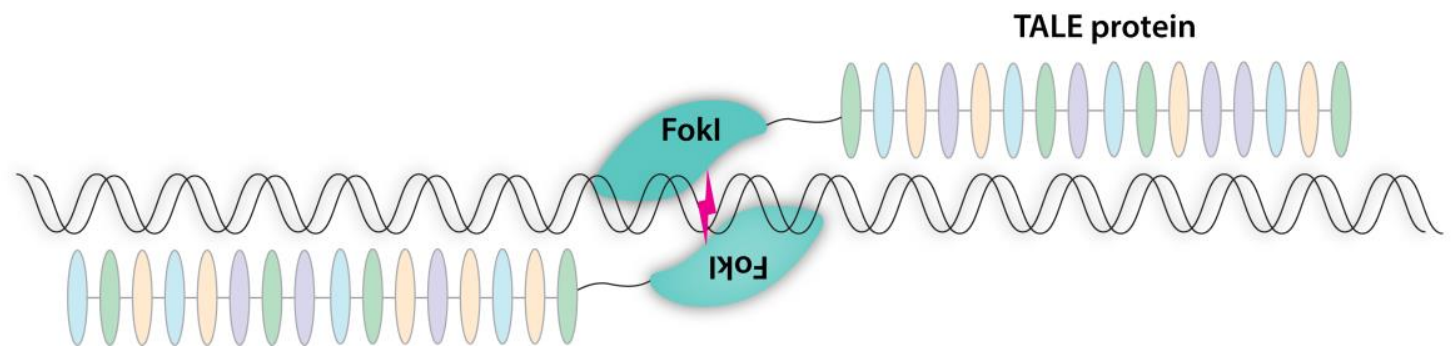

Schematic illustration of reverse genetic mutagenesis approaches based on Fokl endonuclease.

Zinc Finger Nucleases (ZFNs) and Transcription-Activator-Like Effector Nucleases (TALENs) both consist of a DNA binding domain linked to a Fokl endonuclease. Fokl nucleases only induce double strand breaks into the genome if they dimerize. Thus, both ZFNs and TALENs are used in pairs, where proper spacing between the two DNA binding domains is crucial double strand breaks to be induced. a) The DNA binding domain in ZFNs consists of a zinc-finger protein that is engineered to recognize different target sequences. $\mathrm{Many}^{\mathrm{Cis}} \mathrm{His}_{2}$ fingers can be arranged of which the $\alpha$-helix of each finger recognizes 3 to 4 base pairs (Pavletich, Pabo 1991). Zinc-fingers that recognize about 49 out of the 64 existing base pair triplets have been described, however 
specificity is remains problematic. b) Target recognition in TALENs is mediated by the DNA binding domain that consists of tandem repeat units of which each unit specifically binds one nucleotide. One TALEN half-side generally recognizes 16 to 17 nucleotides.

\section{Figure 4:}
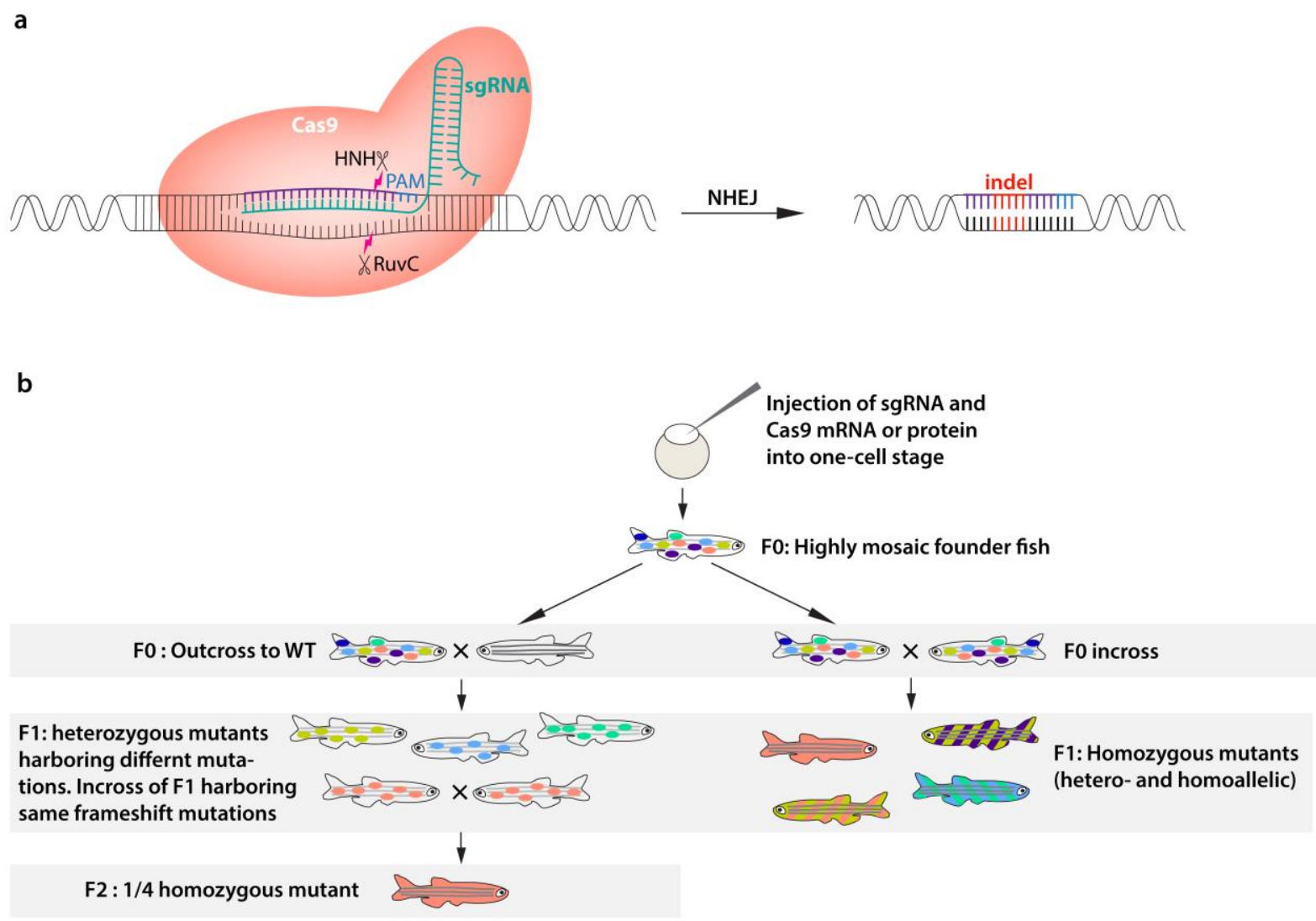

Illustration of a CRISPR/Cas9 complex inducing double strand breaks at the genomic target.

a) The sgRNA targets the Cas9 endonuclease to the genomic DNA, where 20 nucleotides of the sgRNA bind the genomic target by Watson-Crick base pairing. A genomic target consists of 20 nucleotides and an additional 3' NGG protospacer-adjacent motif (PAM) that is required for Cas9 activity. Binding of the sgRNA to the genomic target induces endonuclease activity in the two catalytically active domains RuvC and $\mathrm{HNH}$ of Cas9, inducing site specific nicks between the third and fourth base-pair upstream of the PAM on each strand (Gasiunas et al. 2012; Jinek et al. 2012). A repair mechanism prone to errors ligates the ends of the DNA by non-homologous end joining (NHEJ), resulting in deletions or insertions at the site of the double strand breaks. b) sgRNA with Cas9 mRNA or protein is injected into the one cell stage embryo. F0 founder fish harbor many different mutations (depicted by different colors). Two different breeding schemes are used to generate mutant lines. Mosaic F0 fish can be outcrossed to WT fish to generate a heterozygous F1 generation (left). Two F1 fish harboring the same mutation can be incrossed which results in a partially (1/4) homozygous F2 generation. If high mutation rates are obtained in founder fish, these mosaic F0 fish can directly be incrossed, resulting in a heterogeneous F1 generation (right). This generation needs to be genotyped as both homo- and heteroallelic mutants can be found. Phenotype analysis in homozygous mutants (most likely transheterozygous) can be conducted already in the F1 generation. 